\title{
Type la Supernovae Are Good Standard Candles in the Near Infrared: Evidence From Pairitel
}

\section{Citation}

Wood\#Vasey, W. Michael, Andrew S. Friedman, Joshua S. Bloom, Malcolm Hicken, Maryam Modjaz, Robert P. Kirshner, Dan L. Starr, et al. 2008. “Type la Supernovae Are Good Standard Candles in the Near Infrared: Evidence from PAIRITEL." The Astrophysical Journal 689 (1): 37790. https://doi.org/10.1086/592374.

\section{Permanent link}

http://nrs.harvard.edu/urn-3:HUL.InstRepos:41399736

\section{Terms of Use}

This article was downloaded from Harvard University's DASH repository, and is made available under the terms and conditions applicable to Other Posted Material, as set forth at http:// nrs.harvard.edu/urn-3:HUL.InstRepos:dash.current.terms-of-use\#LAA

\section{Share Your Story}

The Harvard community has made this article openly available. Please share how this access benefits you. Submit a story.

Accessibility 


\title{
TYPE Ia SUPERNOVAE ARE GOOD STANDARD CANDLES IN THE NEAR INFRARED: EVIDENCE FROM PAIRITEL
}

\author{
W. Michael Wood-Vasey, ${ }^{1}$ Andrew S. Friedman, ${ }^{1}$ Joshua S. Bloom, ${ }^{2,3}$ Malcolm Hicken, ${ }^{1}$ Maryam Modjaz, ${ }^{2}$ \\ Robert P. Kirshner, ${ }^{1}$ Dan L. Starr, ${ }^{2}$ Cullen H. Blake, ${ }^{1}$ Emilio E. Falco, ${ }^{1}$ Andrew H. Szentgyorgyi, ${ }^{1}$ \\ Peter Challis, ${ }^{1}$ Stéphane Blondin, ${ }^{1}$ Kaisey S. Mandel, ${ }^{1}$ and Armin Rest ${ }^{4,5}$ \\ Received 2007 November 13; accepted 2008 July 29
}

\begin{abstract}
We have obtained 1087 NIR $\left(J H K_{s}\right)$ measurements of 21 SNe Ia using PAIRITEL, nearly doubling the number of well-sampled NIR SN Ia light curves. These data strengthen the evidence that SNe Ia are excellent standard candles in the NIR, even without correction for optical light-curve shape. We construct fiducial NIR templates for normal SNe Ia from our sample, excluding only the three known peculiar SNe Ia: SN 2005bl, SN 2005hk, and SN $2005 \mathrm{ke}$. The $H$-band absolute magnitudes in this sample of $18 \mathrm{SNe}$ Ia have an intrinsic rms of only 0.15 mag with no correction for light-curve shape. We found a relationship between the $H$-band extinction and optical color excess of $A_{H}=0.2 E(B-V)$. This variation is as small as the scatter in distance modulus measurements currently used for cosmology based on optical light curves after corrections for light-curve shape. Combining the homogeneous PAIRITEL measurements with $23 \mathrm{SNe}$ Ia from the literature, these $41 \mathrm{SNe}$ Ia have standard $H$-band magnitudes with an rms scatter of 0.16 mag. The good match of our sample with the literature sample suggests there are few systematic problems with the photometry. We present a nearby NIR Hubble diagram that shows no correlation of the residuals from the Hubble line with light-curve properties. Future samples that account for optical and NIR lightcurve shapes, absorption, spectroscopic variation, or host-galaxy properties may reveal effective ways to improve the use of SNe Ia as distance indicators. Since systematic errors due to dust absorption in optical bands remain the leading difficulty in the cosmological use of supernovae, the good behavior of SN Ia NIR light curves and their relative insensitivity to reddening make these objects attractive candidates for future cosmological work.
\end{abstract}

Subject headings: distance scale — supernovae: general

Online material: color figures, machine-readable table

\section{INTRODUCTION}

Type Ia supernovae (SNe Ia) observed at optical wavelengths in the rest frame have played a leading role in extragalactic astronomy and cosmology in the past decade. SNe Ia have been the key to measuring the Hubble constant (Freedman et al. 2001; Jha et al. 1999; Riess et al. 2005) and demonstrating cosmic acceleration (Riess et al. 1998; Perlmutter et al. 1999). These discoveries rest on a foundation of photometric and spectroscopic similarities between high- and low-redshift SNe Ia (Hamuy et al. 1996; Riess et al. 1999; Goldhaber et al. 2001; Hook et al. 2005; Blondin et al. 2006; Jha et al. 2006; Conley et al. 2006; Garavini et al. 2007; Garg et al. 2007; Bronder et al. 2007; Foley et al. 2008; Ellis et al. 2008). The history of cosmic expansion has been traced out to redshift $z \sim 1$ ( Tonry et al. 2003; Knop et al. 2003; Barris et al. 2004). The predicted turnover to the matterdominated era and the corresponding deceleration at $z>1$ have been seen in space-based work from the Hubble Space Telescope

\footnotetext{
1 Harvard-Smithsonian Center for Astrophysics, 60 Garden Street, Cambridge, MA 02138; wmwood-vasey@cfa.harvard.edu, afriedman@cfa.harvard.edu, mhicken@cfa.harvard.edu, rkirshner@cfa.harvard.edu, cblake@cfa.harvard .edu, efalco@cfa.harvard.edu, saint@cfa0.cfa.harvard.edu,pchallis@cfa.harvard .edu, sblondin@cfa.harvard.edu,kmandel@cfa.harvard.edu.

${ }^{2}$ Department of Astronomy, University of California, Berkeley, CA 94720; jbloom@astro.berkeley.edu,mmodjaz@astro.berkeley.edu, dstarr@astro.berkeley .edu.

3 Alfred P. Sloan Research Fellow.

4 Cerro Tololo Inter-American Observatory, Colina el Pino S/N, La Serena, Chile.

5 Physics Department, Harvard University, 17 Oxford Street, Cambridge, MA 02138; arest@physics.harvard.edu.
}

(HST) (Riess et al. 2007). Two current projects, ESSENCE (Equation of State: SupErNovae trace Cosmic Expansion; Miknaitis et al. 2007; Wood-Vasey et al. 2007) and SNLS (Supernova Legacy Survey; Astier et al. 2006) seek to constrain the nature of the dark energy responsible for cosmic acceleration by measuring the equation-of-state parameter $w$ with large, homogeneous samples of SNe Ia. ESSENCE and SNLS data are both consistent with dark energy described by a cosmological constant, $w=-1.0$ to better than \pm 0.1 .

These cosmological results are based on measurements of optical emission in the supernova rest frame, but recent work suggests that SNe Ia may be superior distance indicators in the near infrared (NIR), with a narrow distribution of peak $J H K_{s}$ magnitudes and 4-8 times less sensitivity to reddening (Meikle 2000; Krisciunas et al. 2004a, 2007). Reddening and absorption of supernova light by dust in their host galaxies pose the single most vexing systematic question facing SN Ia cosmology (Wang et al. 2006; Conley et al. 2007). It would be very desirable to avoid complications if possible, given the properties of the supernovae. These questions can be approached by building a homogeneous and large set of NIR observations. This paper is a step in that direction.

Early photometric NIR observations of SNe Ia were made by Kirshner et al. (1973); Elias et al. (1981, 1985); Graham et al. (1988); Frogel et al. (1987) with more recent $J H K_{s}$ light curves published by Jha et al. (1999), Hernandez et al. (2000), Valentini et al. (2003), Candia et al. (2003), Krisciunas et al. (2000, 2001, 2003, 2004b, 2004c, 2005, 2006, 2007), Benetti et al. (2004), Elias-Rosa et al. (2006, 2008), Phillips et al. (2006), Pastorello et al. (2007b, 2007a), Prieto et al. (2007), Stritzinger \& Sollerman 
(2007), Wang et al. (2008), Pignata et al. (2008), and Taubenberger et al. (2008). Northern hemisphere work from the Center for Astrophysics (CfA) Supernova Program uses observations from the robotic $1.3 \mathrm{~m}$ Peters Automated Infrared Imaging Telescope (PAIRITEL; Bloom et al. 2006b) at Mount Hopkins, Arizona. The NIR observations are part of a systematic program of supernova observations that includes dense sampling of supernova spectra (Matheson et al. 2008) and concurrent UBVri optical photometry (M. Hicken et al. 2008, in preparation). This paper is a first report on the NIR data on SNe Ia from PAIRITEL. Similar work is underway in the southern hemisphere by the Carnegie Supernova Project using observations at the Las Campanas Observatory in Chile (Freedman 2005; Hamuy et al. 2006). The prospect of large homogeneous data sets coupled with progress in modeling SN Ia NIR light curves (Kasen 2006) raises hopes that SNe Ia, especially in the rest-frame $H$ band, can be developed into the most precise and accurate of cosmological distance probes.

Recent work by Krisciunas et al. (2004a) shows that SNe Ia have a narrow range of luminosity in $J H K_{s}$ at the time of $B$-band maximum light $\left(t_{B \max }\right)$ with smaller scatter than in the $B$ and $V$ bands. Krisciunas et al. (2004a) found no correlation between optical light-curve shape and intrinsic NIR luminosity. The NIR behavior is in sharp contrast with the optical light curves, where a variety of ingenious methods have been devised to reduce the scatter in distance estimates: the $\Delta m_{15}$ method (Hamuy et al. 1996; Phillips et al. 1999; Prieto et al. 2006), the multicolor lightcurve shape method (MLCS/MLCS2k2; Riess et al. 1996, 1998; Jha et al. 2007), the "stretch" method (Perlmutter et al. 1997; Goldhaber et al. 2001), the color-magnitude intercept calibration method (CMAGIC; Wang et al. 2003), the spectral adaptive template method (SALT/SALT2; Guy et al. 2005, 2007; Astier et al. 2006), and SiFTO (Conley et al. 2008). For a sample of $16 \mathrm{SNe}$ Ia observed in NIR bands, Krisciunas et al. (2004a) found an rms of $\sigma_{J}=0.14, \sigma_{H}=0.18$, and $\sigma_{K_{s}}=0.12 \mathrm{mag}$. We set out to construct an independent set of observations to test this remarkable result.

In this paper we present $21 \mathrm{SNe}$ Ia observed with PAIRITEL from 2005 to 2007. The 1087 individual data points in this sample represent the largest homogeneous set of $\mathrm{SNe}$ Ia to date and double the number of NIR SN Ia observations in the literature. Data collection with PAIRITEL is discussed in $\S 2$. Data analysis, including the mosaic creation process and our photometry pipeline, is discussed in $\S 3$. We construct NIR $J H K_{s}$ templates from this new sample as detailed in $\S 4$ and fit these templates to the SN Ia light curves to derive the $J H K_{S}$ magnitudes at the time of $B$-band maximum light. These magnitudes are remarkably uniform, particularly in the $J$ and $H$ bands. Distances are calculated in $\S 5$, and we discuss the standard magnitude of NIR SNe Ia in $\S 6$. We then compare our sample to that compiled in Krisciunas et al. (2004a) as well as to some more recent SNe Ia (Krisciunas et al. 2005, 2006, 2007; Phillips et al. 2006; Pastorello et al. 2007a; Stritzinger \& Sollerman 2007; Wang et al. 2008; EliasRosa et al. 2006, 2008; Pignata et al. 2008; Taubenberger et al. 2008) in $\S 7$ and show that the $J$ and $H$ bands at maximum light, even from this heterogeneous sample, have a small range in absolute magnitude. Our conclusions are summarized in $\S 8$.

\section{DATA}

We present 1087 near-infrared (NIR) measurements with a signal-to-noise ratio $(\mathrm{S} / \mathrm{N})>3$ of 21 nearby $\mathrm{SNe}$ Ia obtained from 2005 to 2007 using the f/13.5 1.3 m PAIRITEL at the F. L. Whipple Observatory on Mount Hopkins in Arizona (Table 1).
Dedicated in 2004 October, PAIRITEL uses the northern telescope of the Two Micron All Sky Survey (2MASS; Skrutskie et al. 2006) together with the 2MASS southern camera. PAIRITEL is a fully automated, robotic telescope with the sequence of observations controlled by an intelligent queue-scheduling database (Bloom et al. 2006b). Two dichroics allow simultaneous observing in $J H K_{s}(1.2,1.6$, and $2.2 \mu \mathrm{m}$; Cohen et al. 2003) with three $256 \times 256$ pixel $\mathrm{HgCdTe}$ NICMOS3 arrays. The image scale of $2^{\prime \prime}$ pixel $^{-1}$ provides a field of view of $8.53^{\prime} \times 8.53^{\prime}$ for each filter. Since the supernova observations are conducted with the instrument that defines the 2MASS photometric system, we use the 2MASS point source catalog (Cutri et al. 2003) to establish the photometric zero points. Images are obtained with standard double-correlated reads with the long (7.8 s) minus short (51 ms) frames in each filter treated as the "raw" frame in the reduction pipeline. The telescope is dithered $\left(<2^{\prime}\right)$ every fourth exposure to aid with reductions.

The CfA Supernova Program used PAIRITEL to follow up $\mathrm{SNe}$ discovered by optical searches at $\delta \gtrsim-30^{\circ}$ with $V \lesssim 18$ mag. These are objects for which we routinely obtain extensive data sets of optical spectra and CCD photometry using other instruments at Mount Hopkins. With 30\% of the time on this dedicated robotic telescope available for supernova observations, we observe approximately three to five SNe per night and obtain host galaxy template reference images for each supernova. The first published PAIRITEL supernova observations were of the unusual core-collapse SN 2005bf ( Tominaga et al. 2005). There have been previous papers that present the data processing from PAIRITEL (Bloom et al. 2006a, 2007; Butler et al. 2006; Yost et al. 2006; Blake et al. 2007; Eisner et al. 2007; Modjaz et al. 2008), but since NIR photometry remains more challenging than optical photometry we present a brief sketch of the data analysis path in the next section.

\section{DATA ANALYSIS}

Data were processed with one of two mosaicking pipelines. For fields dominated by large galaxies we used a pipeline (see Modjaz 2007; Kocevski et al. 2007) that makes use of a bank of existing sky frames. For all other fields, we used the standard pipeline (see $\S 3.1$ ). Both pipelines process the $100-150$ images, each of $7.8 \mathrm{~s}$ duration, by flat correction, dark and sky subtraction, registration, and stacking to create a final mosaicked image in each of the $J H K_{s}$ filters. Mosaicked images typically comprise three images at each dither position, with a mosaicked field of view (FOV) of $\sim 12^{\prime} \times 12^{\prime}$. The raw images are SWarped (Bertin 2005 ) using $0.1^{\prime \prime}$ pixel $^{-1}$ subsampling into final mosaics with $1^{\prime \prime}$ pixel $^{-1}$ sampling. Typical $1800 \mathrm{~s}$ observations (including slew overhead) reach magnitude limits of $\sim 18,17.5$, and $17 \mathrm{mag}$ for $J, H$, and $K_{s}$, respectively.

\subsection{Mosaics}

The PAIRITEL camera has no shutter, so dark current cannot be measured independently, and "skark" background frames include both sky and dark photons. PAIRITEL supernova observations did not include pointings that alternate between the source and the sky, so skark frames were created for each mosaic.

For large host galaxies with angular size $\gtrsim 2^{\prime}$ (in the $12^{\prime} \mathrm{FOV}$ ), host galaxy contamination prevents a reliable calculation of the skark background calculated from a pixel-by-pixel median through the stack of dithered images. Here skark frames are estimated using a median match to an archive of skark frames in relatively empty fields observed on the same night. The archival skark image is selected as close in time to the supernova 
TABLE 1

SN Ia $J H K_{s}$ Peak Magnitudes

\begin{tabular}{|c|c|c|c|c|c|}
\hline $\mathrm{SN}$ & $J_{\text {peak }}{ }^{\mathrm{a}}$ & $H_{\text {peak }}{ }^{\mathrm{a}}$ & $K_{\text {peak }}{ }^{\mathrm{a}}$ & $N_{J}, N_{H}, N_{K}^{\mathrm{b}}$ & References \\
\hline SN 1998bu..... & $11.55 \pm 0.03$ & $11.59 \pm 0.03$ & $11.42 \pm 0.03$ & $18,20,20$ & 2,3 \\
\hline SN 1999cl .............. & $12.80 \pm 0.02$ & $12.77 \pm 0.04$ & $12.58 \pm 0.02$ & $6,5,6$ & 4 \\
\hline 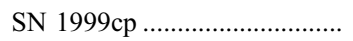 & $14.50 \pm 0.02$ & $14.77 \pm 0.02$ & $14.57 \pm 0.06$ & $2,2,2$ & 4 \\
\hline 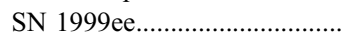 & $14.77 \pm 0.03$ & $15.00 \pm 0.03$ & $\ldots$ & $33,33,0$ & 8 \\
\hline SN 1999ek .................. & $16.10 \pm 0.05$ & $16.08 \pm 0.04$ & $16.54 \pm 0.07$ & $14,15,1$ & 9 \\
\hline 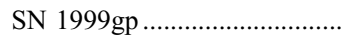 & $17.31 \pm 0.06$ & $17.16 \pm 0.10$ & $16.51 \pm 0.14$ & $3,3,3$ & 16 \\
\hline 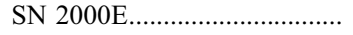 & $13.47 \pm 0.02$ & $13.74 \pm 0.06$ & $13.42 \pm 0.06$ & $16,15,10$ & 6 \\
\hline 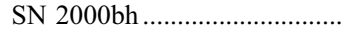 & $16.80 \pm 0.03$ & $16.75 \pm 0.03$ & $16.64 \pm 0.04$ & $20,21,15$ & 8 \\
\hline 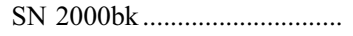 & $17.64 \pm 0.02$ & $17.45 \pm 0.05$ & $\ldots$ & $18,16,0$ & 16 \\
\hline 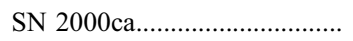 & $16.36 \pm 0.03$ & $16.71 \pm 0.08$ & $\ldots$ & $12,11,0$ & 8 \\
\hline 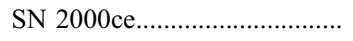 & $16.82 \pm 0.03$ & $16.12 \pm 0.03$ & $15.98 \pm 0.05$ & $3,5,5$ & 16 \\
\hline 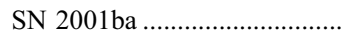 & $16.99 \pm 0.02$ & $17.24 \pm 0.04$ & $17.06 \pm 0.06$ & $15,14,9$ & 8 \\
\hline SN 2001bt...................................... & $15.34 \pm 0.07$ & $15.64 \pm 0.02$ & $15.40 \pm 0.03$ & $21,21,11$ & 9 \\
\hline SN $2001 \mathrm{cn}$ & $15.90 \pm 0.03$ & $15.66 \pm 0.05$ & $15.67 \pm 0.09$ & $19,19,3$ & 9 \\
\hline 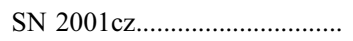 & $15.40 \pm 0.06$ & $15.65 \pm 0.15$ & $15.40 \pm 0.12$ & $12,12,9$ & 9 \\
\hline 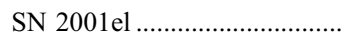 & $12.93 \pm 0.02$ & $12.97 \pm 0.03$ & $12.84 \pm 0.05$ & $33,32,32$ & 7 \\
\hline SN 2002bo ...................................... & $13.68 \pm 0.02$ & $13.82 \pm 0.02$ & $13.88 \pm 0.04$ & $14,14,14$ & 9 \\
\hline 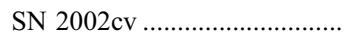 & $14.75 \pm 0.03$ & $14.34 \pm 0.01$ & $13.95 \pm 0.03$ & $17,10,16$ & 5,12 \\
\hline 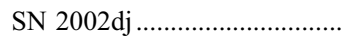 & $14.56 \pm 0.09$ & $14.79 \pm 0.03$ & $14.50 \pm 0.09$ & $21,21,21$ & 15 \\
\hline 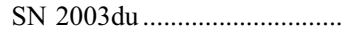 & $14.42 \pm 0.02$ & $14.66 \pm 0.02$ & $14.38 \pm 0.02$ & $6,6,6$ & 14 \\
\hline SN 2003cg & $13.58 \pm 0.08$ & $13.68 \pm 0.10$ & $13.43 \pm 0.01$ & $10,10,10$ & 11 \\
\hline SN 2004S ................................. & $14.95 \pm 0.02$ & $15.04 \pm 0.02$ & $14.87 \pm 0.06$ & $16,17,12$ & 10 \\
\hline 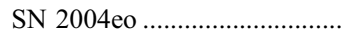 & $15.70 \pm 0.07$ & $15.79 \pm 0.07$ & $15.67 \pm 0.09$ & $11,9,10$ & 13 \\
\hline 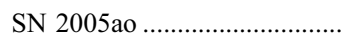 & $17.90 \pm 0.08$ & $17.65 \pm 0.00$ & $16.82 \pm 0.32$ & $6,6,5$ & 1 \\
\hline 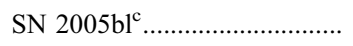 & $17.35 \pm 0.04$ & $17.33 \pm 0.07$ & $17.47 \pm 0.14$ & $15,12,4$ & 17,1 \\
\hline 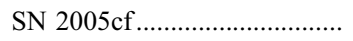 & $13.83 \pm 0.04$ & $13.96 \pm 0.02$ & $13.97 \pm 0.02$ & $17,17,15$ & $1^{\mathrm{d}}$ \\
\hline SN 2005ch & $17.48 \pm 0.06$ & $16.99 \pm 0.05$ & $16.20 \pm 0.14$ & $10,10,8$ & 1 \\
\hline 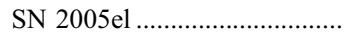 & $15.45 \pm 0.02$ & $15.55 \pm 0.03$ & $15.31 \pm 0.03$ & $29,29,29$ & 1 \\
\hline 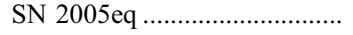 & $16.74 \pm 0.03$ & $17.05 \pm 0.08$ & $16.51 \pm 0.11$ & $30,26,27$ & 1 \\
\hline 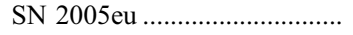 & $17.03 \pm 0.09$ & $17.20 \pm 0.16$ & $16.64 \pm 0.22$ & $22,22,18$ & 1 \\
\hline 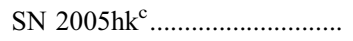 & $15.81 \pm 0.03$ & $15.60 \pm 0.05$ & $15.48 \pm 0.05$ & $18,12,13$ & 1 \\
\hline 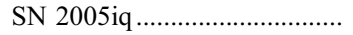 & $17.30 \pm 0.09$ & $17.61 \pm 0.19$ & $\ldots$ & $10,3,0$ & 1 \\
\hline SN $2005 \mathrm{ke}^{\mathrm{c}} \ldots \ldots \ldots \ldots \ldots \ldots$ & $14.18 \pm 0.04$ & $13.98 \pm 0.03$ & $14.03 \pm 0.02$ & $19,18,10$ & 1 \\
\hline 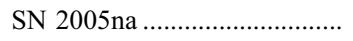 & $17.04 \pm 0.05$ & $16.96 \pm 0.14$ & $16.76 \pm 0.27$ & $24,19,7$ & 1 \\
\hline SN 2006D ...................................... & $14.32 \pm 0.02$ & $14.47 \pm 0.03$ & $14.54 \pm 0.05$ & $22,22,20$ & 1 \\
\hline SN $2006 \mathrm{~N}$ & $16.16 \pm 0.11$ & $15.90 \pm 0.26$ & $15.72 \pm 0.32$ & $14,12,11$ & 1 \\
\hline SN 2006X & $12.86 \pm 0.03$ & $12.92 \pm 0.02$ & $12.74 \pm 0.04$ & $60,66,47$ & $1^{\mathrm{d}}$ \\
\hline 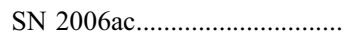 & $16.62 \pm 0.14$ & $16.60 \pm 0.20$ & $16.44 \pm 0.18$ & $20,22,15$ & 1 \\
\hline 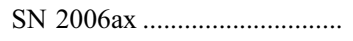 & $15.77 \pm 0.05$ & $16.11 \pm 0.10$ & $15.89 \pm 0.06$ & $17,16,16$ & 1 \\
\hline 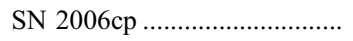 & $17.02 \pm 0.04$ & $16.96 \pm 0.15$ & $16.84 \pm 0.13$ & $5,5,3$ & 1 \\
\hline 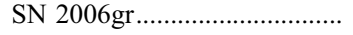 & $17.84 \pm 0.07$ & $17.82 \pm 0.18$ & $17.72 \pm 0.25$ & $6,4,1$ & 1 \\
\hline SN 2006le & $16.15 \pm 0.10$ & $16.38 \pm 0.05$ & $16.05 \pm 0.17$ & $38,32,36$ & 1 \\
\hline 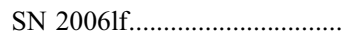 & $15.59 \pm 0.08$ & $15.50 \pm 0.13$ & $15.24 \pm 0.14$ & $30,33,26$ & 1 \\
\hline 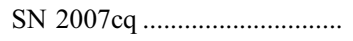 & $16.37 \pm 0.05$ & $16.92 \pm 0.21$ & $\ldots$ & $5,4,0$ & 1 \\
\hline
\end{tabular}

a Magnitudes of the maximum observed magnitude in $J H K_{s}$. Magnitude errors are $1 \sigma$ symmetric errors.

${ }^{\mathrm{b}}$ Number of epochs with $\mathrm{S} / \mathrm{N}>3$ in the $J H K_{s}$ light curves, respectively.

c The fast-declining, unusual SN 2005bl, SN 2005hk, and SN 2005ke were excluded from the template generation. When fit with the $H$-band template they are clearly rejected as outliers with $\chi^{2} /$ dof $>3$. See Fig. 3 for a visual comparison of the $H$-band light curves of these dromedary SNe Ia compared with the $H$-band template.

${ }^{d}$ Because we had data from PAIRITEL on these SNe Ia we did not take advantage of the photometry of SN $2005 \mathrm{cf}$ provided in Pastorello et al. (2007b) or that of SN 2006X provided in Wang et al. (2008).

REFERENCES.-(1) PAIRITEL photometry; this paper; (2) Jha et al. 1999; (3) Hernandez et al. 2000; (4) Krisciunas et al. 2000; (5) Di Paola et al. 2002; (6) Valentini et al. 2003; (7) Krisciunas et al. 2003; (8) Krisciunas et al. 2004a; (9) Krisciunas et al. 2004b; (10) Krisciunas et al. 2007; (11) Elias-Rosa et al. 2006; (12) Elias-Rosa et al. 2008; (13) Pastorello et al. 2007a; (14) Stanishev et al. 2007; (15) Pignata et al. 2008; (16) Krisciunas et al. 2001; (17) Taubenberger et al. 2008.

observations as possible, with a median matched in a $50 \times 50$ pixel box, known to have stable pixel properties, in the lower left quadrant of the array. After the dark-plus-sky frame is subtracted, images are mosaicked using the drizzle technique (Fruchter \& Hook 2002).

For well-isolated supernovae with host galaxies of angular size $\lesssim 2^{\prime}$ (in a $12^{\prime}$ FOV), skark frames can be constructed from the science images themselves. This construction is done by applying a cubic $B$-spline spline fit to a complete time series of sky values in each pixel for that observation. Since the fitting characteristics of the spline curve vary by filter, elevation, and weather, parameters are adjusted to best fit each observation and filter. The pixels with sources are masked out using object masks generated from SExtractor catalogs extracted from the raw frames. The pipeline replaces each masked pixel's flux value with a simplere "median skark" pixel value that is the sum of the median flux value of each frame and a median over time of the pixel's deviation from this median frame value. 


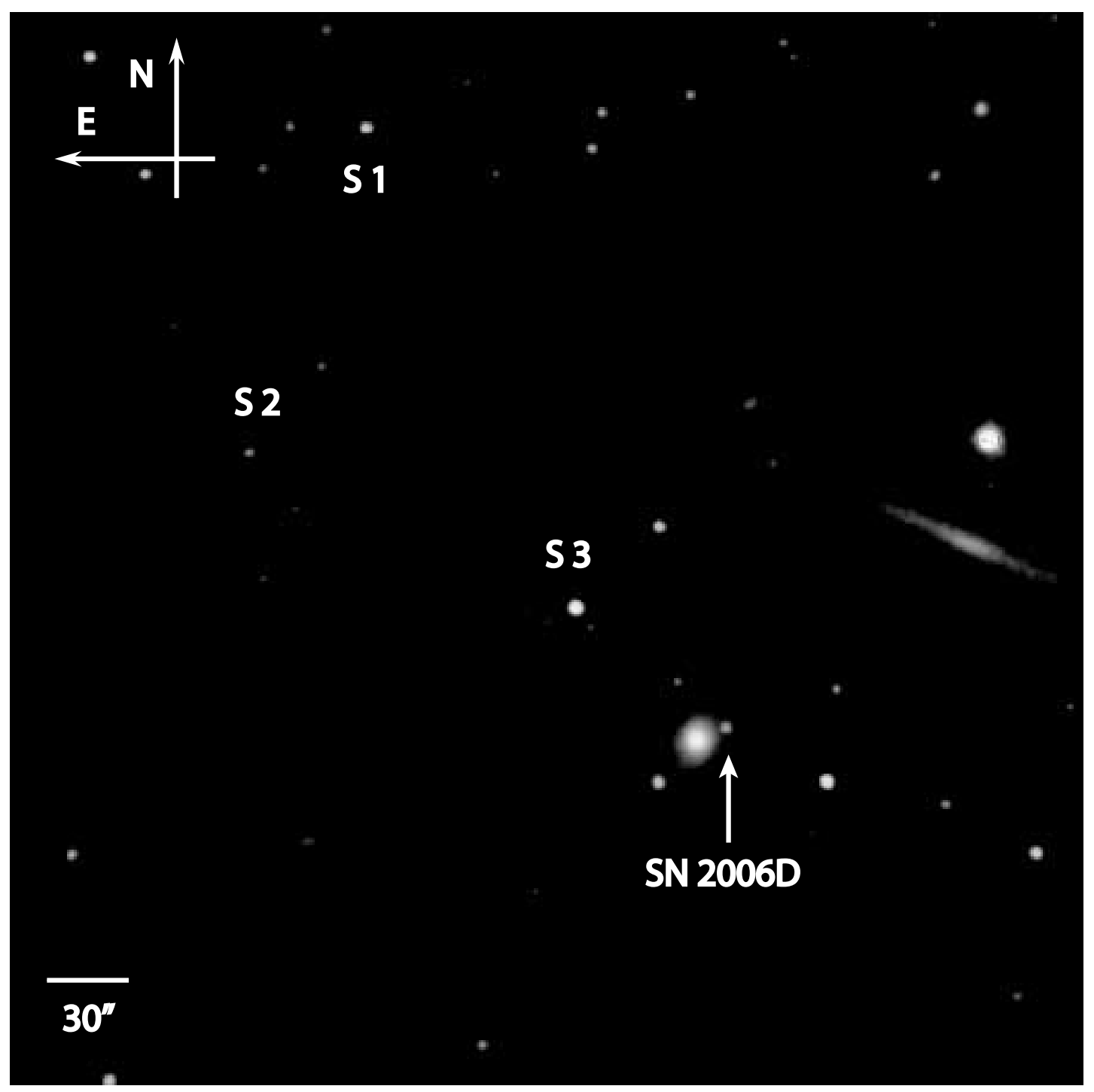

FIG. 1.-PAIRITEL $J H K_{s}$ composite color image of SN 2006D at a week past maximum light when the SN Ia had NIR magnitudes of $(J, H, K)(16.1,14.8,15.0)$. The image shown is $7.5^{\prime} \times 7.5^{\prime}$ in size. There are $~ 30$ 2MASS stars in this field that were used for the photometric calibration. The S1, S2, and S3 labels indicate three representative 2MASS stars with $J H K_{s}$ magnitudes of S1: (14.11, 13.76, 13.71), S2: (16.02, 15.335, 14.830), and S3: (13.23, 12.61, 12.41). [See the electronic edition of the Journal for a color version of this figure.]

The spline fit parameters define a function that tracks the time variation of the skark background in each of the $256 \times 256$ pixels over the duration of the dither pattern. We construct a skark image at the time of each $7.8 \mathrm{~s}$ science exposure by calculating the skark value in each pixel at this time and subtracting that skark image from the science image. For cases in which the galaxy occupies a small fraction of the field, this process improves the sky subtraction. This standard pipeline works better than simply taking the pixel-by-pixel median through the dithered (unregistered) stack of science images because it samples the sky variation on timescales that are shorter than the total exposure time. Mosaicked images are constructed using SWarp (Bertin 2005). We use SWarp with a simple BILINEAR sampling because such a kernel is most suited to our undersampled raw images.

Most of our objects were reduced with the standard processing code. Only SN 2005cf, SN 2005ke, SN 2005na, and SN 2006X required the large galaxy approach. For both methods, bad-pixel masks and flat fields were created from archival images. Figure 1 shows a final $J H K_{S}$ color mosaic created in the standard processing mode.

\subsection{Photometry}

Mosaicked images were fed to the photometry pipeline we have used in the ESSENCE and SuperMACHO projects (Rest et al. 2005; Garg et al. 2007; Miknaitis et al. 2007). This pipeline

TABLE 2

Light Curve Table

\begin{tabular}{|c|c|c|c|c|}
\hline SN & MJD & Passband & $\operatorname{Flux}_{25}{ }^{\mathrm{a}}$ & Flux err 25 \\
\hline 2005ао......................... & $53,440.4157$ & $H$ & 600.2491 & 3.5737 \\
\hline 2005аo.............................. & $53,456.4300$ & $H$ & 437.9836 & 3.6405 \\
\hline 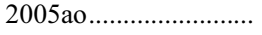 & $53,464.3872$ & $H$ & 339.2386 & 128.0434 \\
\hline 2005аo............................. & $53,466.3879$ & $H$ & 345.1047 & 90.6351 \\
\hline 2005ао............................. & $53,467.3847$ & $H$ & 561.7854 & 3.7594 \\
\hline
\end{tabular}

Note.-Table 2 is published in its entirety in the electronic edition of the Astrophysical Journal. A portion is shown here for guidance regarding its form and content.

${ }^{a}$ Fluxes are expressed normalized to a zero point of 25 ; i.e., the calibrated 2 MASS magnitude is mag $=-2.5 \log _{10}$ (flux $)+25$. 


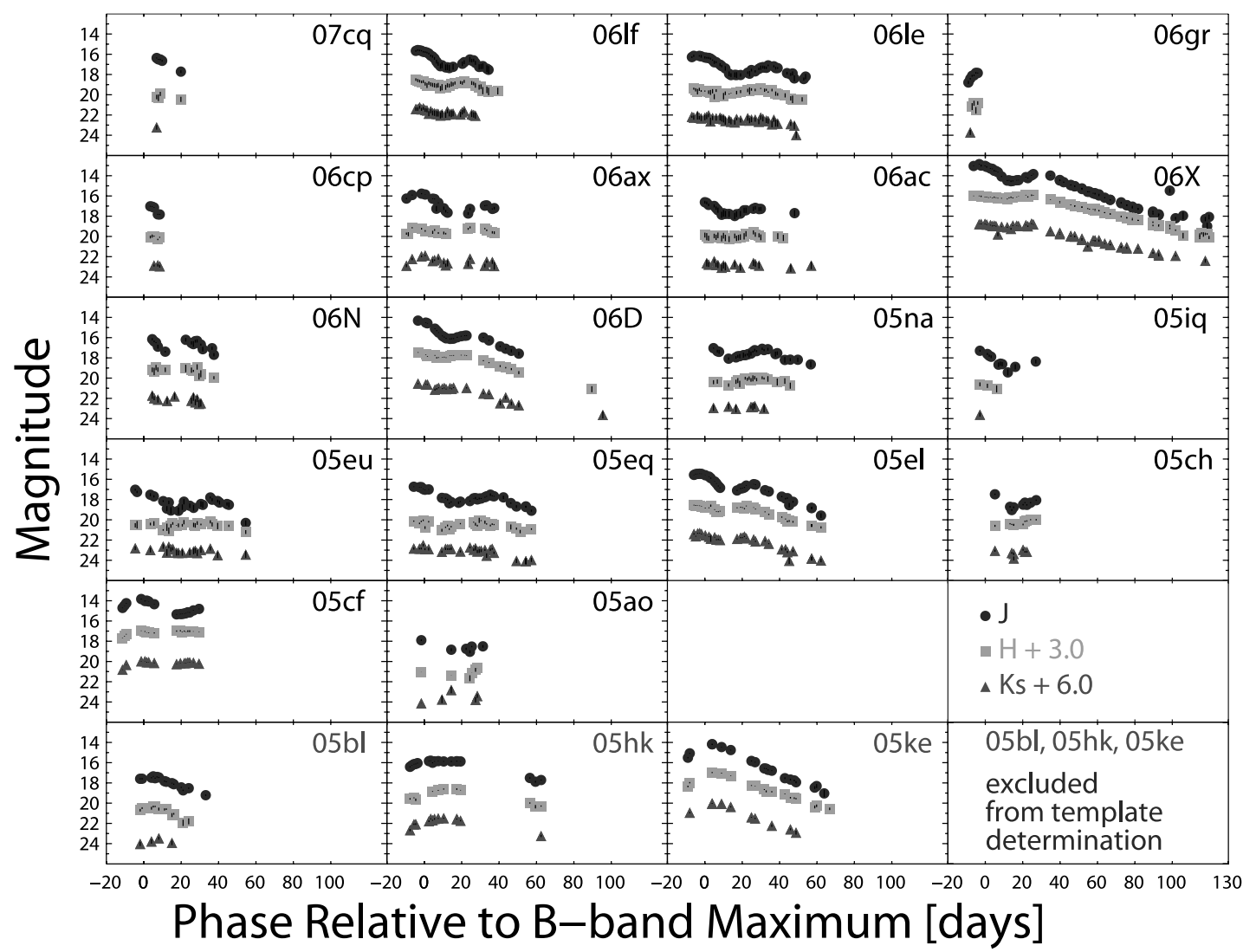

FIG. 2.-PAIRITEL NIR light curves of the $21 \mathrm{SNe}$ Ia presented in this paper. There are 18 normal SNe Ia plus 3 fast-declining or unusual SNe Ia: SN $2005 \mathrm{bl}$, SN $2005 \mathrm{hk}$, and SN 2005ke. These SNe Ia are displayed on their own row at the bottom of the plot. [See the electronic edition of the Journal for a color version of this figure.]

determines the photon noise for each pixel based on the sky noise in the mosaic images, registers the images for a supernova to a common reference frame, and performs point-spread function (PSF) photometry using DoPHOT (Schechter et al. 1993). The 2MASS catalog (Cutri et al. 2003) is the natural astrometric and photometric reference system for these observations. In a typical $12^{\prime} \times 12^{\prime}$ field of view, there were 10-100 2MASS stars in each filter. These stars were sufficient to calibrate the images to the 2MASS system $J H K_{S}$ firmly enough that the underlying uncertainty in the 2MASS system, of about $3 \%$, is the dominant error in the photometric calibrations for the light curves presented here. Cohen et al. (2003) describe the 2MASS $J H K_{s}$ filter system in detail and Leggett et al. (2006) provide color transformations to put observations with the 2MASS filter system on other widely used photometric systems.

Final reference images were taken for each galaxy after the supernova had faded. DoPHOT photometry on the images was used for those SNe Ia that were clearly separated from their host galaxy and had little underlying contaminating light (SN 2005ao, SN 2005cf, SN 2005el, SN 2005hk, SN 2005ke, SN 2005eu, SN 2005iq, SN 2005na, SN 2006N, and SN 2006X). The standard background annulus subtraction used by DoPHOT to estimate the local sky was sufficient to remove any remaining contaminating galaxy light. In every case, the contribution from underlying galaxy light was less than $10 \%$ of the SN Ia light at maximum. Seeing at PAIRITEL is limited by the dome seeing and remains relatively constant from $1.8^{\prime \prime}$ to $2.0^{\prime \prime}$. The good effect of this mediocre dome seeing is that the contaminating galaxy light within the PSF is very nearly constant.

We used subtraction-based photometry (following Miknaitis et al. 2007) for SNe Ia that were not clearly separated from their

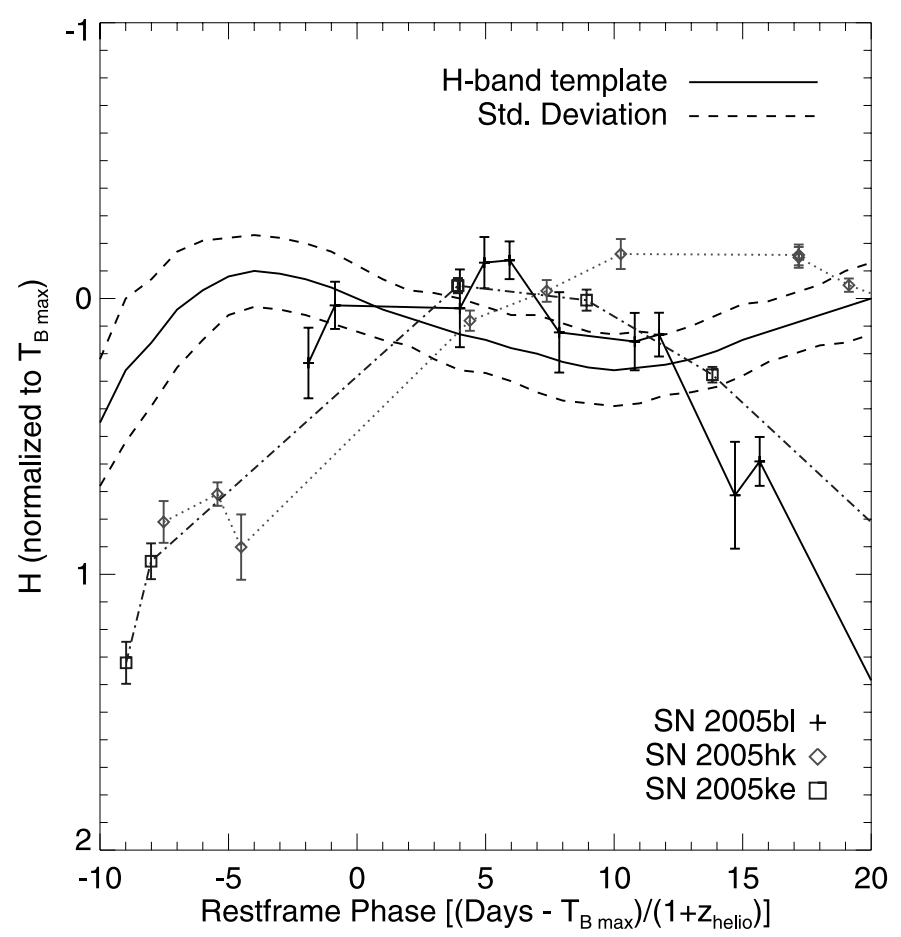

FIG. 3.- SN 2005bl, SN 2005hk, and SN 2005ke were observed as part of the PAIRITEL campaign but were excluded from the construction of the template because they were known to be unusual SNe Ia. The $H$-band template we have constructed for this paper is valid from -10 to +20 rest-frame days from $B$-band maximum light. We compare the $H$-band light curves of these unusual SNe Ia with the normal SN Ia $H$-band template to demonstrate the clarity with which these unusual supernovae can be distinguished from the normal SNe Ia used in the Hubble diagram. [See the electronic edition of the Journal for a color version of this figure.] 
TABLE 3

Apparent Standard $H$-Band Magnitudes of Type Ia Supernovae

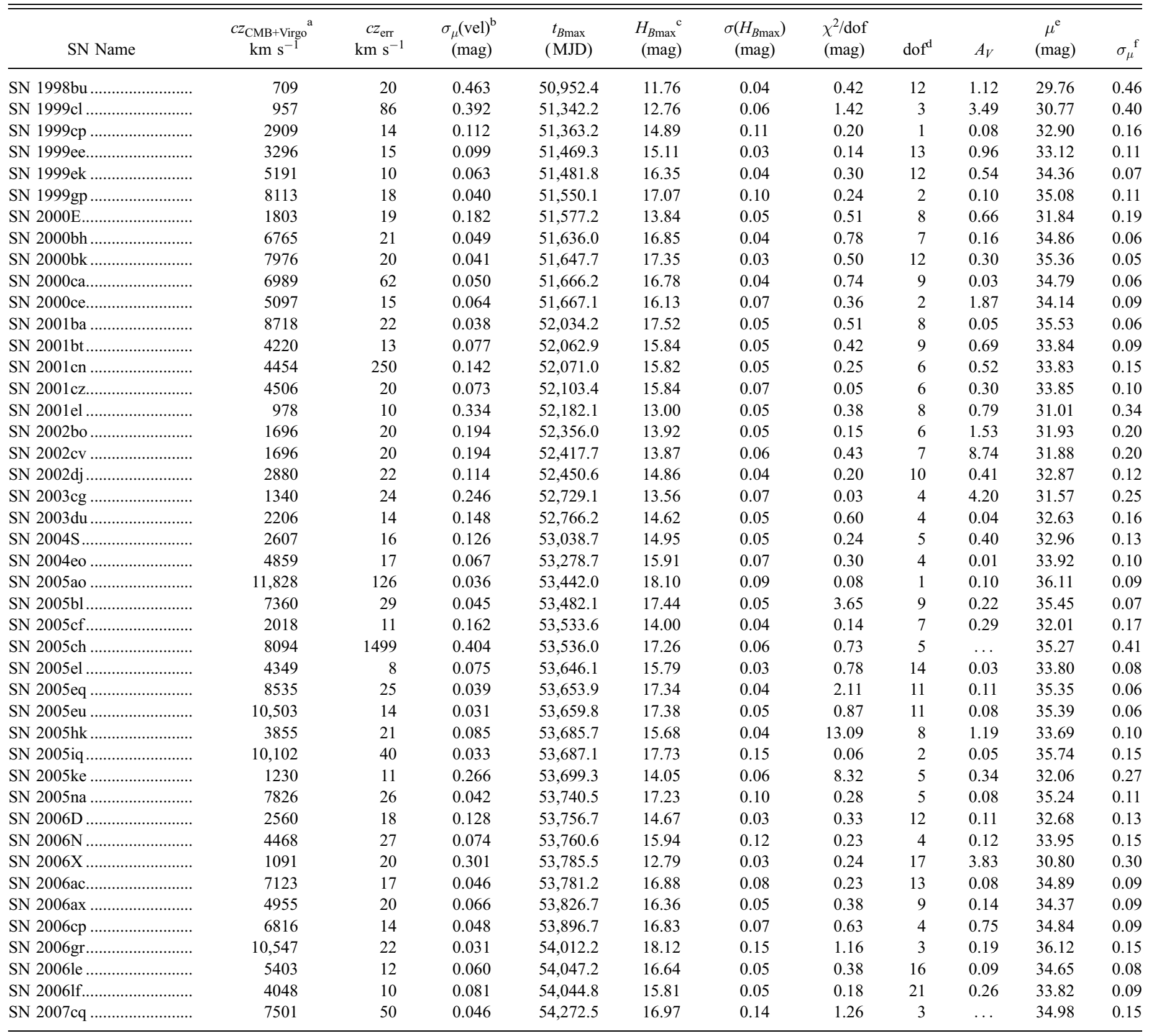

${ }^{a}$ Redshift of host galaxies as corrected to CMB frame with the additional correction of Virgo member NGC 4501 (host of SN 1999cl) from its observed CMBcorrected recession velocity $\left(2281 \mathrm{~km} \mathrm{~s}^{-1}\right)$ to the mean recession velocity of Virgo $\left(957 \mathrm{~km} \mathrm{~s}^{-1}\right)$.

${ }^{b}$ A peculiar velocity of $150 \mathrm{~km} \mathrm{~s}^{-1}$ and the individual redshift measurement uncertainty of col. (2) was converted into an equivalent distance modulus uncertainty using Eq. (1) .

${ }^{c}$ An H-band extinction correction of $A_{H}=0.06 A_{V}$ has been applied to the magnitudes obtained by fitting to the template. See $\S 6$ for details

${ }^{\mathrm{d}}$ dof: The number of degrees of freedom is the number of $H$-band data points minus 1 for the overall offset fit parameter, $H_{B \max }$.

e Assuming $H_{0}=72 \mathrm{~km} \mathrm{~s}^{-1} \mathrm{Mpc}^{-1}$ and thus $M_{H}=-18.07 \pm 0.03$ mag.

${ }^{\mathrm{f}}$ Quadrature sum of peculiar velocity distance modulus uncertainty (col. [4]) and fit uncertainty (col. [7]).

host galaxy. The NN2 method of Barris et al. (2005) was employed (as used in Miknaitis et al. 2007) by subtracting all $N \times$ $(N-1) / 2$ unique pairs of images to minimize the sensitivity to subtraction errors. Because our PAIRITEL data are not critically sampled, the reliability of the PAIRITEL image subtraction is not as good as the subtractions in Miknaitis et al. (2007). Our photometric pipeline automatically detected and screened out subtractions with significant residual flux in known stars, leaving us with fewer high-quality light-curve points than would have been obtained in the case of perfect subtractions. For the supernovae for which the underlying galaxy contribution was a small fraction of the supernova light, direct photometry on the unsubtracted images proved preferable.

Photometry was extracted from either the unsubtracted or the subtracted images by forcing DoPHOT to measure the PSFweighted flux of the object at a fixed position. This position was 
determined in the $J$-band difference images that had a $\mathrm{S} / \mathrm{N}>5$. This average position was used for fixed-position DoPHOT photometry of each image of a SN Ia.

Flux measurements were calibrated to the 2MASS system by using photometric solutions to the 2MASS catalogs (Cutri et al. 2003). For the difference images the calibrated zero point from the template was used, with suitable correction for the convolution of the template image as detailed by Miknaitis et al. (2007). Our NIR PAIRITEL light curves for the $21 \mathrm{SNe}$ Ia presented in this paper are given in Table 2 and shown in Figure 2.

\section{NIR TEMPLATE}

We constructed $J H K_{s}$ templates with NIR light curves from PAIRITEL, using our own optical CCD observations (M. Hicken et al. 2008 , in preparation) to establish the time of $B$-band maximum, $t_{B \max }$.

In constructing the templates, we excluded the three fastdeclining SNe Ia from our overall sample of 21 SNe Ia: SN 2005bl, SN 2005hk, and SN 2005ke. SN 2005bl has an optical fast decline rate (Taubenberger et al. 2008) similar to the archetypal fast-decliner, SN 1991bg (Filippenko et al. 1992). SN 2005hk is known to be an unusual SN Ia (Phillips et al. 2007; Sahu et al. 2008). SN 2005ke is a 1991 bg-like SN Ia with possible circumstellar interaction (Patat et al. 2005; Immler et al. 2006). SN 2005bl, SN 2005hk, and SN 2005ke exhibit only one infrared hump instead of the two exhibited by normal SNe Ia. The $J H K_{s}$ maximum for these "dromedary" light curves occurs after $B$-band maximum light, unlike normal "bactrian" $\mathrm{SNe}$ Ia for which the first NIR maximum occurs 3-5 rest-frame days before $B$-band maximum light. We compare our SN Ia $H$-band template to SN 2005bl, SN 2005ke, and SN 2005hk in Figure 3. Because we have excluded these three objects, our template does not extend to $1991 \mathrm{bg}$-like SNe Ia or other unusual SNe Ia. This seems a reasonable approach while the data set is small. Unusual supernovae can be identified from a NIR light curve alone, or they can be identified from their optical spectra and light curves which we obtain as a matter of course. In any case, we defer incorporating these unusual objects into a more comprehensive treatment of NIR light curves until the available database of near-infrared SN Ia light curves is more fully populated.

We applied the $K_{s}$-corrections of Krisciunas et al. (2004b) to the NIR light curves based on the heliocentric redshift. The NIR light curves for the remaining $18 \mathrm{SNe}$ Ia were registered to a common phase by subtracting $t_{B \max }$ and accounting for time dilation based on the heliocentric redshift, $z_{\text {helio }}$ (Leibundgut et al. 1996; Goldhaber et al. 2001). Unlike Krisciunas et al. (2004a) we do not further adjust this phase by the optical lightcurve width parameter. We found that compensating for the lightcurve width gave no improvement in the $H$-band template fits (which is the focus of this paper) or in the resulting dispersion of absolute magnitudes (see $\S 6$ ) determined between -10 and +20 days in phase. However, the position of the $J$-band second NIR maximum is variable and may be related to intrinsic luminosity. In larger data sets and in more detailed investigations of the $J$ band it will be worth exploring whether a width-correction parameter would produce a more effective template for fitting the second maximum at later times.

Between $[-10,+30]$ days, where we have excellent sampling, we constructed our light curve templates by an unweighted averaging of the points from this initial set of standardized light curves into 1 day bins. No outlier rejection was used in this process. The SN Ia light curves were then registered to a common magnitude scale by fitting each to this zeroth-iteration template and determining the maximum magnitude at $B$-band maximum
TABLE 4

JHK Absolute Magnitudes of SNe Ia at $t_{B \max }$

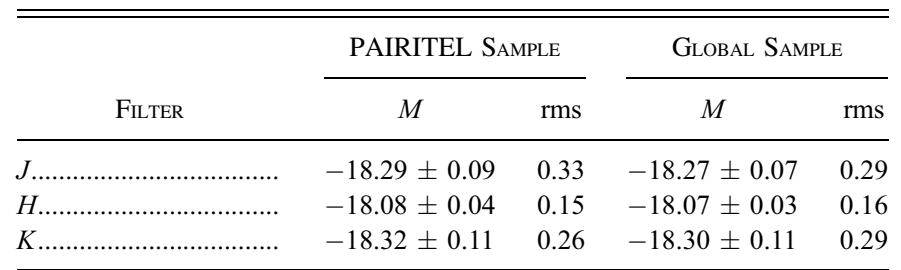

Notes.-The $J$-band template shows significant variation as a function of optical light-curve width. The numbers derived above are for the average template as shown in Fig. 4. The $K$-band data from PAIRITEL is only useful for nine new SNe Ia and does not significantly improve on the results of Krisciunas et al. (2004a).

light $\left(\mathrm{IR}_{B \max }\right)$. The fitting process is insensitive to the determination of the initial $I_{B \max }$ because this process is repeated with each iteration and the memory of the first guess is completely erased after the second iteration. At late times ( $>30$ days), we had fewer points from which to construct the template, but it was clear that the SNe Ia were consistent with a linear fit. For our templates after +30 days we adopted linear templates based on a fit to SN Ia data at these late times with a forced connection to the binned value at 30 days. The complete template was then smoothed twice with a boxcar of length 5 days. The smoothing has very little effect near maximum light because of our dense sampling at those epochs. Varying the smoothing length between 1 and 5 days did not substantially affect the template.

To refine this template, we determined the magnitude value at $t_{B \max }$ that minimized the $\chi^{2}$ of the template versus the data over the span from $[-10,+20]$ days. These new magnitude offsets were then used to seed the process above where we had previously used zero for the $\mathrm{IR}_{B \max }$ values. The procedure was iterated twice more to construct final values at $t_{B \max }$ for each SN Ia, and the final template was constructed based on these values. We found that three iterations were sufficient to reach convergence. The uncertainty in the template is the standard deviation of the residuals of the SN Ia light-curve points around the mean template in a moving 5 day window.

We also explored using a simple linear interpolation on 1 day sampling and found that the binned template and the linearly interpolated templates agreed to within hundredths of a magnitude in this region near peak. We take this as a strong indication that we have reached limit of intrinsic variability of SNe Ia in the construction of this template, since the effective use of 5 times more data in the binned approach yielded the same template as the linear interpolation, which only uses $\sim 20 \%$ of the available data.

This template construction procedure was performed for each of the $J H K_{s}$ NIR passbands. For our data, the $H$-band light curve was observed to exhibit small scatter from $[-10,+30]$ days with a particularly tight distribution from $[-10,+20]$ days. The $J$-band aggregate light curve has small scatter about the template from $[-10,+10]$ days but begins to show variations in the time and flux of the secondary maximum for individual supernovae. The $K_{s}$-band data from our PAIRITEL observations is not as good as the $J$ - and $H$-band data due to increased sky background in $K_{s}$. Our template in the $K_{s}$ band shows significantly more scatter with fewer objects. We draw no firm conclusions in this paper with regard to $K_{s}$-band SN Ia light curves.

The unusual cases that were excluded from the template construction are easily seen to be far from the $H$-band template. Fitting SN 2005bl, SN 2005hk, or SN 2005ke with the PAIRITEL SN Ia $H$-band template results in a sufficiently bad $\chi^{2} /$ dof $(>3)$ 

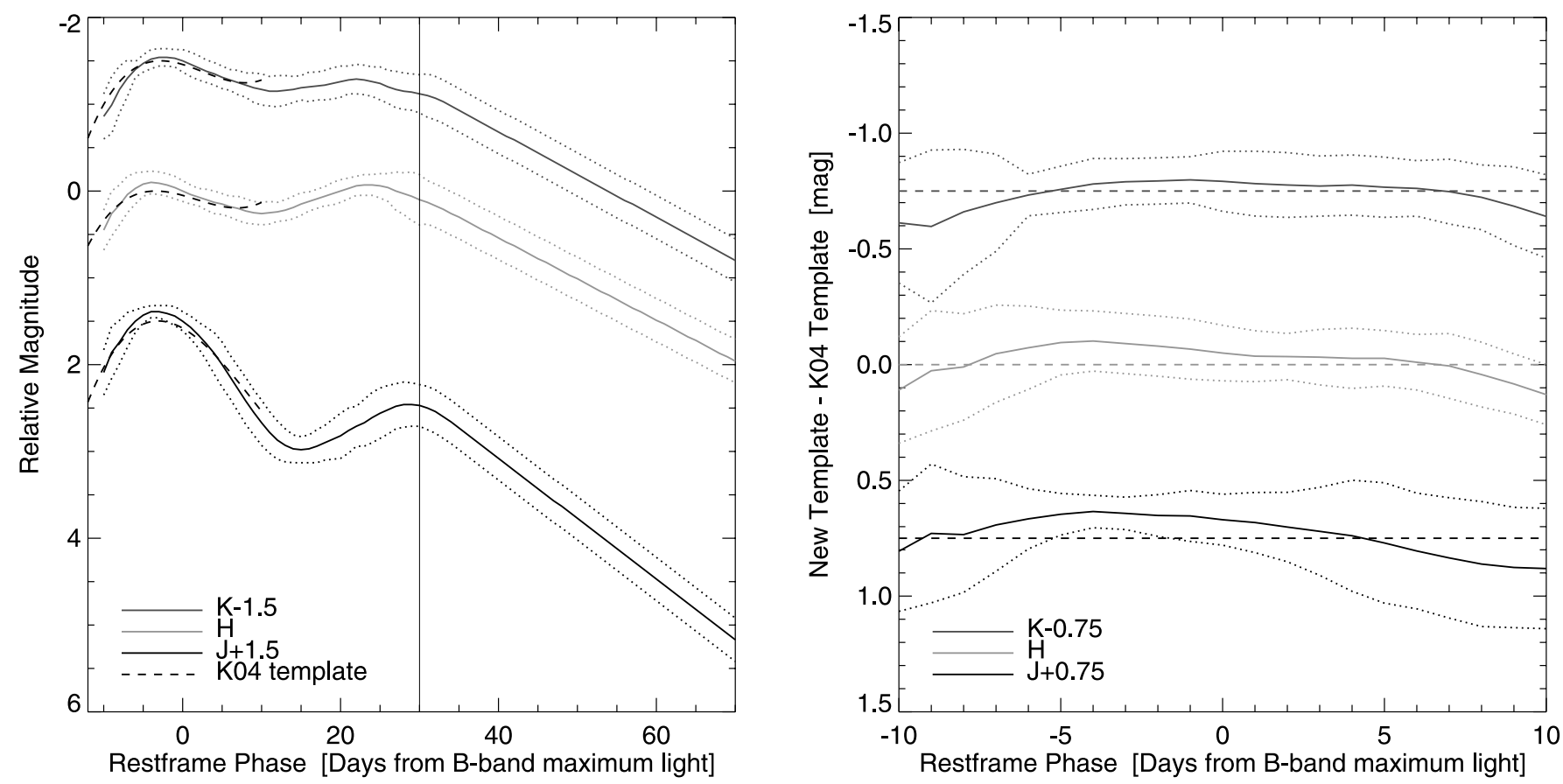

FIG. 4.- SN Ia $J H K_{s}$ templates. The $1 \sigma$ uncertainties (dashed lines) are based on the sample variance within a 5 day moving window of the SNe Ia with respect to the

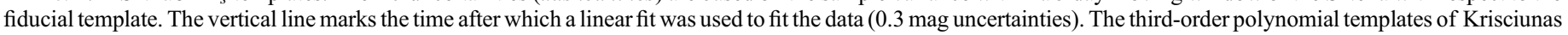

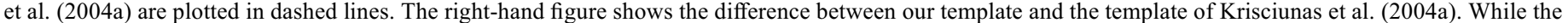

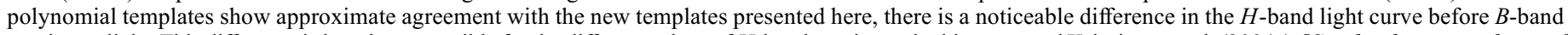
maximum light. This difference is largely responsible for the different values of $H$-band maximum in this paper and Krisciunas et al. (2004a). [See the electronic edition of the Journal for a color version of this figure.]

(see Table 3) that these SNe Ia would be excluded based on that criterion alone.

\section{GALAXY REDSHIFTS}

Recession velocities for our SNe Ia are those of the identified host galaxies in the NASA/IPAC Extragalactic Database (NED). ${ }^{6}$ We use the quoted velocity with respect to the Virgo infall model of Mould et al. (2000). For SNe Ia within $3000 \mathrm{~km} \mathrm{~s}^{-1}$ we used available information on distances to the galaxies to minimize our sensitivity to the details of the local flows. We used the values of Krisciunas et al. (2004a, 2004b) for SN 1998bu and SN 2002bo and Wang et al. (2008) for SN 2006X. After accounting for these $\mathrm{SNe}$ Ia, we were left with three SNe Ia within $3000 \mathrm{~km} \mathrm{~s}^{-1}$ for which we used the Mould et al. (2000) values, SN 2003du, SN 2005cf, and SN 2006D. Outside of $3000 \mathrm{~km} \mathrm{~s}^{-1}$ differences among various flow models are not significant. One supernova, SN 1999cl, occurred in a Virgo Cluster member galaxy, NGC 4501, so we used the Virgo Cluster redshift instead of the recession velocity of NGC 4501. We assumed a peculiar velocity of $\sigma_{\mathrm{vel}}=$ $150 \mathrm{~km} \mathrm{~s}^{-1}$ (Radburn-Smith et al. 2004) and the redshift measurement uncertainty for each host galaxy as reported by NED. These uncertainties were converted into a distance modulus uncertainty, $\sigma_{\mu}(\mathrm{vel})$, using equation (1):

$$
\sigma_{\mu}(\mathrm{vel})=\frac{5}{z \ln 10} \sqrt{\left(\sigma_{\mathrm{vel}} / c\right)^{2}+\sigma_{z}^{2}}
$$

This is valid for a smooth cosmology that resembles any currently allowed cosmological expansion model. Values for $\sigma_{\mu}(\mathrm{vel})$ for the $\mathrm{SNe}$ Ia considered in this paper are given in column (12) of Table 3.
We confirmed the NED heliocentric redshift values with the heliocentric galaxy redshifts derived from spectra of the hosts as observed by the CfA Supernova Program ${ }^{7}$ and measured using the galaxy redshift code RVSAO (Kurtz \& Mink 1998). All were consistent with the NED values within $200 \mathrm{~km} \mathrm{~s}^{-1}$. For SN 2007cq, no NED redshift was available for the host galaxy so we used the value from the CfA galaxy spectrum as the heliocentric velocity and then used the Mould et al. (2000) model conversion provided by NED to express this redshift in the $\mathrm{CMB}+$ Virgo infall frame.

\section{DISTANCE MODULI AND THE STANDARD NIR BRIGHTNESS OF SNe Ia}

The most uniform behavior of our SN Ia light curves is in the $H$-band, and that band is where we focus our analysis. In the most simplistic treatment, we take the $H_{B \max }$ for each SN Ia to have a standard value. Then the distance modulus is simply

$$
\mu=H_{B \max }-M_{H_{B \max }}
$$

where $M_{H_{B \max }}$ is the absolute $H$-band magnitude of a SN Ia at $B$-band maximum light. The numerical value for the absolute magnitude of SNe Ia in $H$ is separate from the question of how well the $H$-band magnitudes trace cosmic expansion.

When we combined the results for the PAIRITEL SNe Ia with our analysis of the literature SNe Ia in $\S 7$ we found that an $H$-band extinction correction of $A_{H}=0.19 E(B-V)$ best minimized the correlation between Hubble-diagram residual and $E(B-V)$ as measured by MLCS2k2 and applied that correction to the apparent magnitudes computed from fitting to our $H$-band template. While the trend was not formally statistically significant, because the most highly extinguished SNe Ia are also 
TABLE 5

SN Ia $J H K_{s}$ Templates

\begin{tabular}{|c|c|c|c|c|c|c|}
\hline Phase & $J$ Magnitude & Uncertainty & $H$ Magnitude & Uncertainty & $K_{s}$ Magnitude & Uncertainty \\
\hline-10.00 & 0.59 & 0.26 & 0.45 & 0.23 & 0.64 & 0.26 \\
\hline-9.00 & 0.36 & 0.30 & 0.26 & 0.26 & 0.51 & 0.33 \\
\hline-8.00 & 0.24 & 0.25 & 0.16 & 0.23 & 0.33 & 0.27 \\
\hline-7.00 & 0.10 & 0.20 & 0.04 & 0.21 & 0.20 & 0.21 \\
\hline-6.00 & 0.00 & 0.13 & -0.03 & 0.18 & 0.10 & 0.09 \\
\hline-5.00 & -0.07 & 0.09 & -0.08 & 0.14 & 0.03 & 0.10 \\
\hline-4.00 & -0.11 & 0.07 & -0.10 & 0.13 & -0.02 & 0.11 \\
\hline-3.00 & -0.11 & 0.07 & -0.09 & 0.13 & -0.04 & 0.10 \\
\hline-2.00 & -0.09 & 0.09 & -0.07 & 0.13 & -0.04 & 0.10 \\
\hline-1.00 & -0.06 & 0.11 & -0.04 & 0.13 & -0.03 & 0.10 \\
\hline 0.00 & 0.00 & 0.11 & 0.00 & 0.12 & 0.00 & 0.13 \\
\hline 1.00 & 0.07 & 0.13 & 0.04 & 0.11 & 0.04 & 0.14 \\
\hline 2.00 & 0.16 & 0.15 & 0.07 & 0.10 & 0.08 & 0.14 \\
\hline 3.00 & 0.26 & 0.19 & 0.10 & 0.12 & 0.12 & 0.13 \\
\hline 4.00 & 0.37 & 0.24 & 0.13 & 0.13 & 0.15 & 0.13 \\
\hline 5.00 & 0.50 & 0.26 & 0.15 & 0.12 & 0.19 & 0.13 \\
\hline 6.00 & 0.64 & 0.25 & 0.18 & 0.12 & 0.22 & 0.12 \\
\hline 7.00 & 0.78 & 0.26 & 0.20 & 0.14 & 0.25 & 0.14 \\
\hline 8.00 & 0.92 & 0.27 & 0.23 & 0.14 & 0.28 & 0.14 \\
\hline 9.00 & 1.05 & 0.26 & 0.25 & 0.13 & 0.31 & 0.17 \\
\hline 10.00 & 1.17 & 0.26 & 0.26 & 0.13 & 0.33 & 0.18 \\
\hline 11.00 & 1.28 & 0.24 & 0.25 & 0.13 & 0.35 & 0.17 \\
\hline 12.00 & 1.37 & 0.22 & 0.24 & 0.11 & 0.35 & 0.18 \\
\hline 13.00 & 1.43 & 0.19 & 0.22 & 0.12 & 0.34 & 0.17 \\
\hline 14.00 & 1.47 & 0.16 & 0.19 & 0.13 & 0.33 & 0.15 \\
\hline 15.00 & 1.48 & 0.15 & 0.15 & 0.13 & 0.31 & 0.14 \\
\hline 16.00 & 1.47 & 0.16 & 0.12 & 0.11 & 0.30 & 0.17 \\
\hline 17.00 & 1.44 & 0.19 & 0.09 & 0.11 & 0.29 & 0.16 \\
\hline 18.00 & 1.40 & 0.20 & 0.06 & 0.11 & 0.28 & 0.17 \\
\hline 19.00 & 1.36 & 0.23 & 0.03 & 0.13 & 0.26 & 0.18 \\
\hline 20.00 & 1.32 & 0.26 & 0.00 & 0.13 & 0.24 & 0.18 \\
\hline 21.00 & 1.26 & 0.26 & -0.03 & 0.12 & 0.22 & 0.16 \\
\hline 22.00 & 1.21 & 0.23 & -0.06 & 0.11 & 0.21 & 0.17 \\
\hline 23.00 & 1.17 & 0.27 & -0.07 & 0.12 & 0.22 & 0.17 \\
\hline 24.00 & 1.11 & 0.29 & -0.07 & 0.12 & 0.24 & 0.17 \\
\hline 25.00 & 1.06 & 0.29 & -0.06 & 0.14 & 0.26 & 0.19 \\
\hline 26.00 & 1.02 & 0.28 & -0.04 & 0.16 & 0.30 & 0.19 \\
\hline 27.00 & 0.98 & 0.26 & 0.00 & 0.22 & 0.33 & 0.21 \\
\hline 28.00 & 0.96 & 0.26 & 0.03 & 0.24 & 0.35 & 0.21 \\
\hline 29.00 & 0.96 & 0.25 & 0.06 & 0.28 & 0.36 & 0.21 \\
\hline 30.00 & 0.97 & 0.24 & 0.10 & 0.29 & 0.38 & 0.22 \\
\hline 31.00 & 1.00 & 0.25 & 0.13 & 0.25 & 0.40 & 0.25 \\
\hline 32.00 & 1.04 & 0.25 & 0.17 & 0.25 & 0.43 & 0.25 \\
\hline 33.00 & 1.10 & 0.25 & 0.21 & 0.25 & 0.47 & 0.25 \\
\hline 34.00 & 1.16 & 0.25 & 0.26 & 0.25 & 0.52 & 0.25 \\
\hline 35.00 & 1.23 & 0.25 & 0.30 & 0.25 & 0.57 & 0.25 \\
\hline 36.00 & 1.30 & 0.25 & 0.35 & 0.25 & 0.62 & 0.25 \\
\hline 37.00 & 1.37 & 0.25 & 0.40 & 0.25 & 0.67 & 0.25 \\
\hline 38.00 & 1.44 & 0.25 & 0.45 & 0.25 & 0.72 & 0.25 \\
\hline 39.00 & 1.51 & 0.25 & 0.49 & 0.25 & 0.77 & 0.25 \\
\hline 40.00 & 1.58 & 0.25 & 0.54 & 0.25 & 0.82 & 0.25 \\
\hline 50.00 & 2.27 & 0.25 & 1.01 & 0.25 & 1.31 & 0.25 \\
\hline 60.00 & 2.97 & 0.25 & 1.49 & 0.25 & 1.80 & 0.25 \\
\hline 70.00 & 3.67 & 0.25 & 1.96 & 0.25 & 2.30 & 0.25 \\
\hline 80.00 & 4.37 & 0.25 & 2.44 & 0.25 & 2.79 & 0.25 \\
\hline
\end{tabular}

NoтE.-The templates are linear fits from +35 to +80 days with $\left(J, H, K_{s}\right)$ slopes of $(0.0625,0.0470$, $0.425)$ mag day $^{-1}$.

the ones that are at the lowest recession velocity and have the most uncertain distances, it was consistent with direct comparison of extinction for SNe Ia in the same galaxy. Two of the SNe Ia in our sample, SN 2002bo and SN 2002cv, were both in NGC 3190 and had significant extinction. When we assumed that each of these SNe Ia had the same intrinsic brightness, we found the same relationship between $H$-band extinction and optical color excess of $A_{H}=0.2 E(B-V)$. While quantitatively uncertain, this $R_{H} \sim 0.2$ corresponds to an equivalent $R_{V}=1.2$, which is in qualitative agreement with previous work on observed supernova color-luminosity behavior (see Perlmutter et al. 1999; Knop et al. 2003; Guy et al. 2005, 2007; Conley et al. 


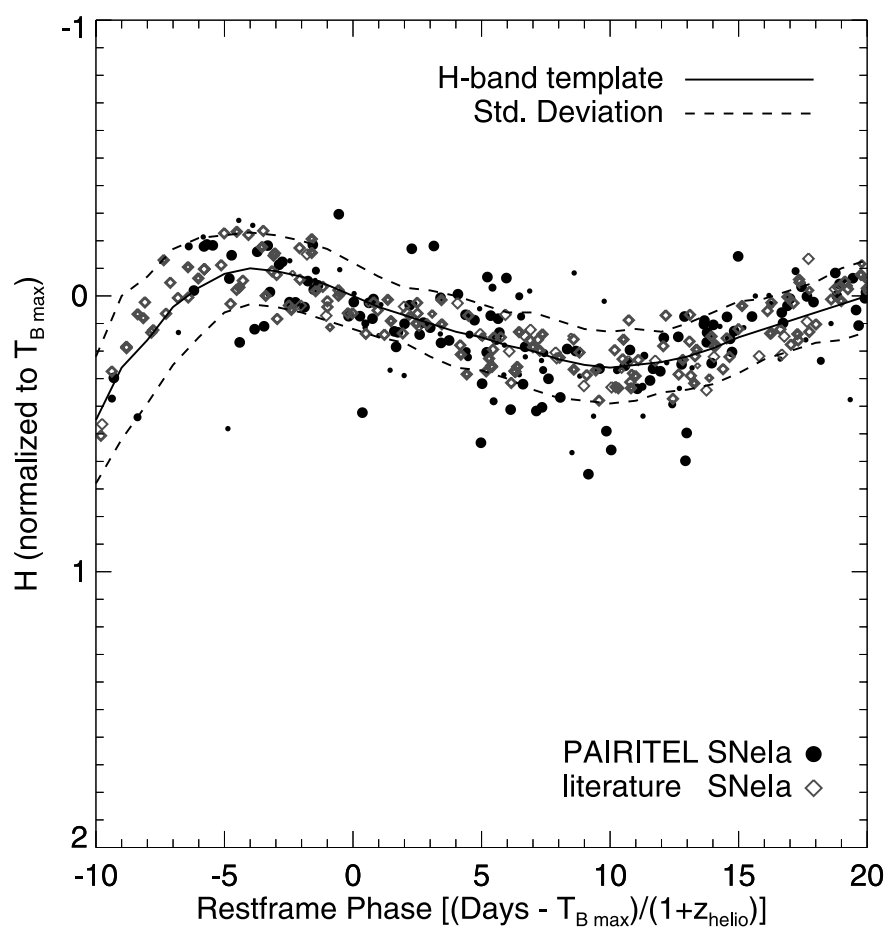

FIG. 5.-NIR $H$-band template based on all of the SNe Ia considered in this paper (excluding the dromedary SNe Ia shown in Fig. 3) together with the constituent SN Ia observations. Model uncertainties are indicated by dashed lines as in Fig. 4. For clarity data error bars are not displayed. Instead points are scaled by their S/N (small: $10 \geq \mathrm{S} / \mathrm{N}>3$; medium: $20 \geq \mathrm{S} / \mathrm{N}>10$; large: $\mathrm{S} / \mathrm{N}>20$ ). [See the electronic edition of the Journal for a color version of this figure.]

2007). Resolving this mysterious behavior of supernova colors, host galaxy dust, and apparent luminosity is currently the major challenge in SN Ia cosmology.

The set of redshifts, apparent magnitudes, and accompanying uncertainties from $\S 5$ were compared to a $\Lambda$ CDM concordance cosmology $\left(\Omega_{M}, \Omega_{\Lambda}, w\right)=(0.27,0.73,-1)$. We solve for the absolute mean brightness of a SN Ia in $H$-band at $B$-band maximum light by taking the weighted mean of the differences of the apparent magnitudes and the $\Lambda \mathrm{CDM}$ distance moduli (the exact cosmology does not quantitatively affect the results at these redshifts):

$M_{H_{B \max }}=\sum_{i=1}^{N} \frac{H_{B \max }^{i}-\mu^{i}}{\sigma_{H_{B \max }^{i 2}}+\sigma_{\mu}^{i}(\mathrm{vel})^{2}} / \sum_{i=1}^{N} \frac{1}{\sigma_{H_{B \max }^{i 2}}+\sigma_{\mu}^{i}(\mathrm{vel})^{2}}$.

Assuming an $H_{0}$ of $72 \mathrm{~km} \mathrm{~s}^{-1} \mathrm{Mpc}^{-1}$ (Freedman et al. 2001; Spergel et al. 2003, 2007), we find $M_{H_{B \max }}=-18.08 \pm 0.04$ with an rms of 0.15 mag for the fiducial $H$-band magnitude at $B$-band maximum light (Table 4). A similar analysis for the $J$-band magnitude at $B$-band maximum light finds $M_{J_{\max }}=$ $-18.29 \pm 0.09$ with an rms of $0.33 \mathrm{mag}$. These values are for the new SNe Ia presented here.

For a cosmological fit one would marginalize over the combination

$$
\begin{aligned}
\mathcal{M}_{H_{B \max }} & =M_{H_{B \max }}-5 \log _{10} h_{0}+15+5 \log _{10} c \\
\sigma_{\mathcal{M}_{H_{B \max }}} & =5 \frac{\sigma_{h_{0}}}{h_{0}} \log _{10} e
\end{aligned}
$$

where $h=H_{0} /\left(100 \mathrm{~km} \mathrm{~s}^{-1} \mathrm{Mpc}^{-1}\right)$ and $c$ is the speed of light in vacuum in kilometers per second. The marginalization in our case is trivial because we are assuming a fixed $\Lambda \mathrm{CDM}$ cosmology.

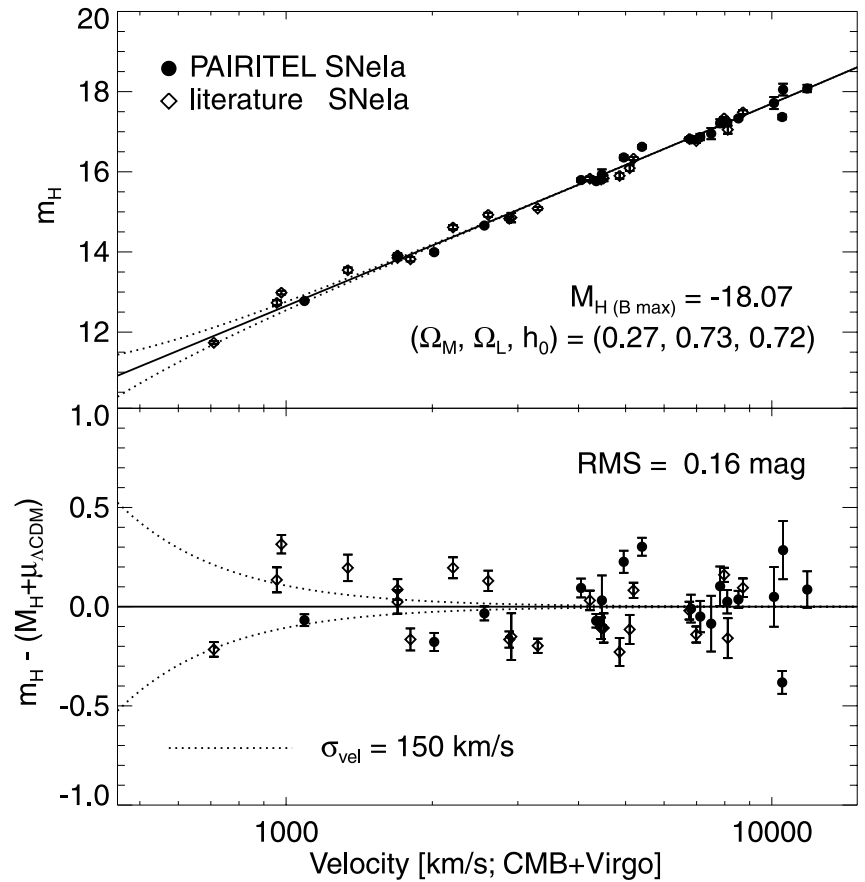

FIG. 6. $-H$-band SN Ia Hubble diagram ( $\mu_{H}$ vs. $z$ and residual vs. $z$ ). The rms dispersion is $0.15 \mathrm{mag}$. SNe Ia from the literature are shown as open diamonds, while the new SNe Ia from this paper are shown as filled circles. The error bars are the fit uncertainties from the fit to the $H$-band template (col. [7] of Table 3). The reduced Hubble constant, $h_{0}$, is $h_{0}=H_{0} / 100 \mathrm{~km} \mathrm{~s}^{-1} \mathrm{Mpc}^{-1}$. [See the electronic edition of the Journal for a color version of this figure.]

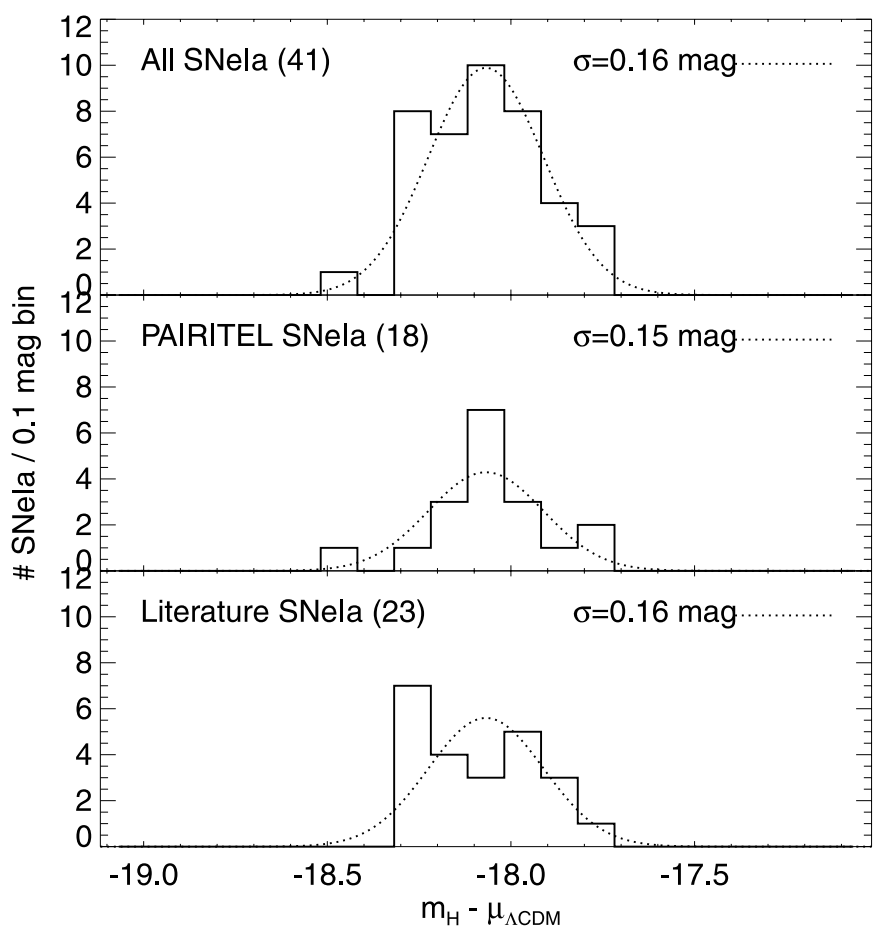

FIG. 7.- Histogram of the derived absolute magnitudes $M_{H_{B \max }}$ based on a $\Lambda \mathrm{CDM}$ cosmology with $H_{0}=72 \mathrm{~km} \mathrm{~s}^{-1} \mathrm{Mpc}^{-1}$ for all SNe Ia (top) along with histograms for the PAIRITEL sample (middle) and the literature SNe Ia (bottom). Overlying each histogram is a Gaussian with the rms width of the given sample (dotted line) normalized to the number of SNe Ia in each plot. 

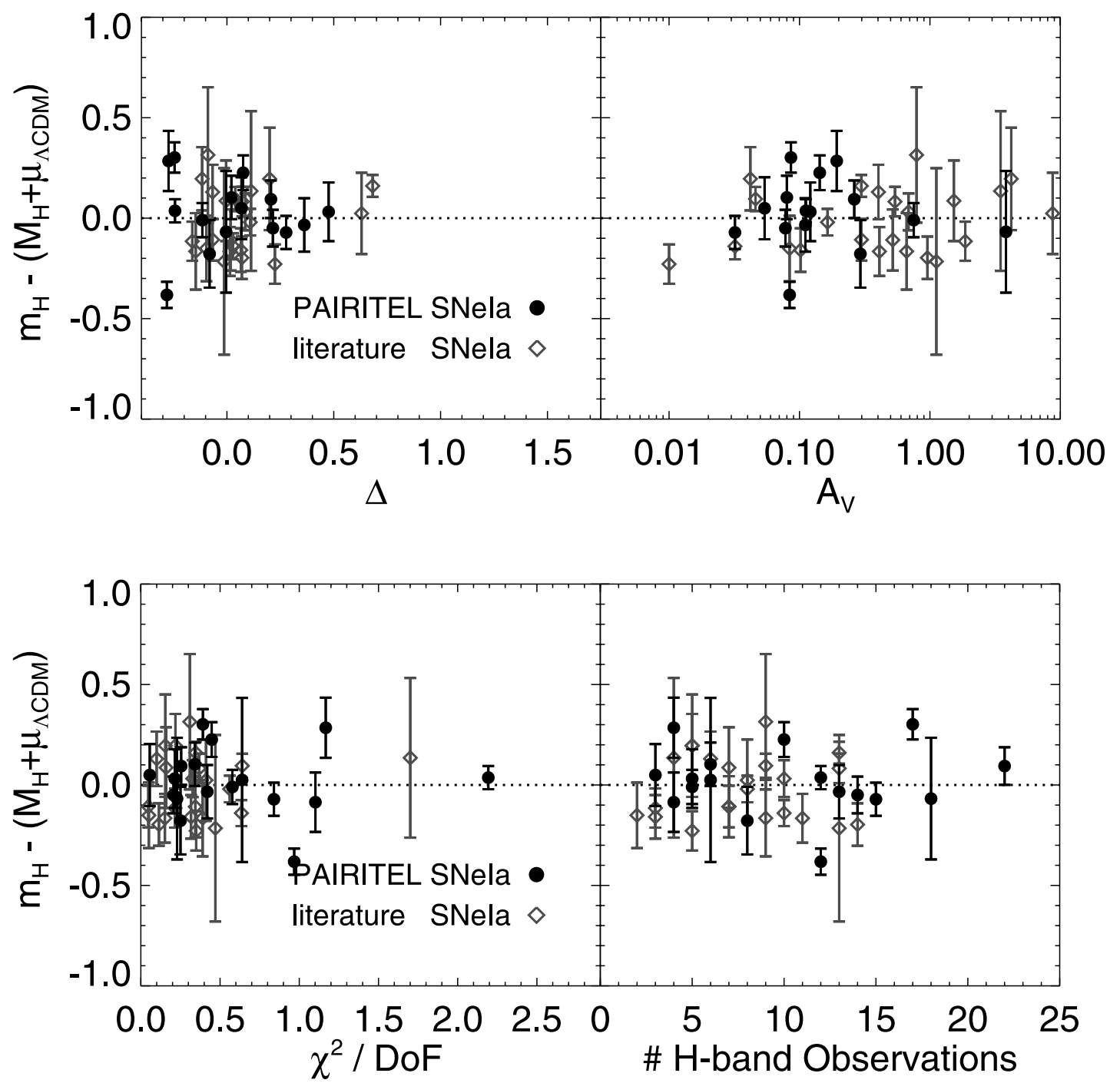

FIg. 8.- Hubble diagram residuals. Top: The Hubble diagram residuals of the extinction-corrected $m_{H}$ as a function of the light-curve shape parameter, $\Delta$, and the measured optical extinction, $A_{V}$. Both plots are entirely consistent with no dependence of Hubble diagram residual on $\Delta$ after the extinction correction of $A_{H}=0.06 A_{V}$. Bottom: Hubble diagram residuals as a function of $\chi^{2}$ dof and the number of $H$-band light-curve points $($ dof $=$ number of light-curve points -1$)$. These plots demonstrate that the residuals are not sensitive to the formal $\chi^{2} /$ dof of the fit and are reliable even with just a few data points. The large number of points with $\chi^{2} /$ dof $<1$ implies that we have likely overestimated the uncertainties in our fiducial template or in our photometric observations. Unlike in Fig. 6, the error bars shown here represent both the model uncertainties ( Table 3, col. [7]) and the peculiar velocity $\left(150 \mathrm{~km} \mathrm{~s}^{-1}\right.$ ) (Table 3, col. [4]) added in quadrature ( Table 3, col. [12]) to better allow for a relative comparison of the deviation. There is a mild correlation between the size of the error bars and $A_{V}$ because the SNe Ia with large $A_{V}$ also tend to be nearby $\left(z<2000 \mathrm{~km} \mathrm{~s}{ }^{-1}\right)$ due to selection effects against finding highly extinguished supernovae at larger distances. [See the electronic edition of the Journal for a color version of this figure.]

This assumption allows us to propagate a $H_{0}$ uncertainty of $10 \%$ directly through equation (6) to determine our global uncertainty in $M_{H_{B \max }}$. The mean value of $M_{H_{B \max }}$ is unchanged by the marginalization, but the total uncertainty is increased to \pm 0.22 . The uncertainty in $H_{0}$ dominates the overall uncertainty of $M_{H_{B \max }}$.

Small variation in the NIR absolute magnitudes of SNe Ia is an important result that was first found by Krisciunas et al. (2004a). Here we have an independent confirmation of this result from a homogeneous set of well-sampled SNe Ia light curves observed with PAIRITEL. In the next section we expand that data set by joining the PAIRITEL sample with SNe Ia from the literature.

\section{COMPARISON WITH LITERATURE SNe Ia}

We joined our PAIRITEL sample with $23 \mathrm{SNe}$ Ia from the literature (see Table 3) extending back to SN 1998bu (earlier observations of supernovae with earlier detectors were not included in this study). When creating the template including the literature SNe Ia, we excluded SN 1999ac because it was classified as an unusual SN Ia (Phillips et al. 2006). The PAIRITEL SNe Ia listed in $\S 4$ were similarly excluded from the global templategeneration. The literature $\mathrm{SNe}$ Ia that were published on the Persson et al. (1998) system were converted to the 2MASS system using the conversion equations of Carpenter (2001). These corrections were typically on the order of $0.01 \mathrm{mag}$. We generated a new set of NIR templates following the same prescription as in $\S 4$ and found that the full sample of SNe Ia was just as well behaved as the PAIRITEL sample. This is a tribute to the care of the observers and the clear definition of the 2MASS photometric system. The NIR SN Ia templates and associated standard deviations for this combined sample are shown in Figure 4 and given in Table 5. The small scatter of the $H$-band light curves from $[-10,+20]$ days and the $J$-band light curves from $[-10,+10]$ days was preserved in the heterogeneous sample. Figure 5 shows the tight scatter of the $H$-band SN Ia light-curve data points around 
the fiducial template. Overplotted in Figure 4 are the NIR templates of Krisciunas et al. (2004a). There is general agreement but noticeable systematic discrepancy in the $H$-band template before maximum light. Together with the difference in definition of quoted $H$-band maximum (in this paper we refer to $M_{H}$ at $B$-band maximum light, whereas Krisciunas et al. [2004a] quote the observed $H$-band magnitude at $H$-band maximum light), this disagreement in the $H$-band templates explains the bulk of the 0.3 mag difference between the quoted $M_{H}$ values in these respective works.

We present the $H$-band apparent magnitudes from this global template in Table 3. The comparison of these apparent magnitudes with the expected luminosity distance relation of a $\Lambda \mathrm{CDM}$ cosmology, $\left(\Omega_{M}, \Omega_{\Lambda}, h_{0}, w\right)=(0.23,0.77,0.72,-1)$, is shown in Figure 6. We find a global $H$-band rms of 0.16 mag around an absolute magnitude of $M_{H}=-18.07 \pm 0.03$ (see Fig. 7). A similar analysis for $J$-band finds similar agreement with the PAIRITEL-only values with a global value of $M_{J}=$ $-18.27 \pm 0.07$ with an rms of $0.29 \mathrm{mag}$. The $K_{s}$-band data from PAIRITEL is more limited and only adds nine normal $\mathrm{SNe}$ Ia to the literature. The updated global values including these new SNe Ia against the global template as generated for $J$ and $H$ are $M_{K}=-18.30 \pm 0.11$ with an rms of 0.29 mag. To check the quality of the light-curve fits and to see whether the fits are robust, we plot the $H$-band residuals with respect to the $\chi^{2} /$ dof and degrees of freedom of the fits to the $H$-band template in Figure 8 . Figure 8 demonstrates that after an extinction correction of $A_{H}=0.19 E(B-V)$, there is no correlation in the $H$-band residuals with the light-curve shape parameter, $\Delta$, or extinction, $A_{V}$, as measured from the optical light curves by MLCS2k2 (Jha et al. 2007).

To reduce the effects of uncertainties in the local flow, we also calculated the rms for only the $28 \mathrm{SNe}$ Ia with a CMB+Virgo infall model velocity of $>2000 \mathrm{~km} \mathrm{~s}^{-1}$. We found an rms of 0.15 mag for this sample. Previous work by Radburn-Smith et al. (2004) and Neill et al. (2007) found that SN Ia distances were more consistent with the Hubble law when a local flow model was incorporated. Future work from larger samples of nearby SNe Ia in the NIR will allow for the investigation of both the local flow and the absolute NIR magnitudes of SNe Ia from integrated analyses with more sophisticated flow models of the local universe.

\section{CONCLUSIONS}

We have constructed improved NIR templates to find accurate luminosity distances to $\mathrm{SNe}$ Ia using the $H$-band light curve from $[-10,+20]$ days after $B$-band maximum light. Within the photometric and local-flow uncertainties, the distribution about a best-fit $\Lambda C D M$ model is consistent with no intrinsic dispersion of our $H$-band template fit magnitude values. Even without accounting for peculiar velocity uncertainties, the rms scatter of 0.15 mag from these uncorrected inferred luminosity distance moduli is as small as corrected distances from optical-based methods. No correction for light-curve shape has been made here and the treatment of dust extinction is relatively limited in its extrapolation over a factor of 2 in wavelength. It is quite plausible that using information on the NIR and optical lightcurve shapes, dust absorption as fit from the optical to the infrared, spectroscopic variation, or host-galaxy properties will result in even smaller scatter and better distance determinations.

The Krisciunas et al. (2004a) result was determined from an inhomogeneous sample of 16 light curves. This paper improves on that work by providing a homogeneous sample of 18
PAIRITEL SN Ia light curves that more than doubles the number of independent photometric observations in the literature. With our ongoing program at Mount Hopkins adding 10-15 SNe Ia per year, PAIRITEL will continue building an extensive nearby training set of ground-based NIR SNe Ia. While we observe all the SNe Ia that meet our criteria, objects in the Hubble flow observed with adequate integration times to beat down the measurement errors will be the most helpful.

In this paper we considered reddening values derived from optical data, and found a relationship of apparent $A_{H}=0.06 A_{V}$ between the absolute $H$-band magnitude and the optical reddening. However, recent work on the highly extinguished SN $2002 \mathrm{cv}$ (Elias-Rosa et al. 2008) shows that reddening corrections will be necessary for some highly absorbed SNe Ia in the NIR. Reddening uncertainties derived from optical data alone currently represent the most significant systematic error affecting SN Ia luminosity distance measurements. Simulations by Krisciunas et al. (2007) demonstrate major improvements to be gained from the addition of NIR data. Krisciunas et al. (2007) show that simulated distance modulus errors are improved by factors of 2.7 and 3.5 by adding $J$, and $J H K_{s}$, respectively, to $U B V R I$ data. Reducing systematics due to reddening are crucial to future spacebased SN Ia surveys which will be large enough to avoid limitations from sample size. In that case, improving the constraints on cosmological parameters will be limited by systematics. We will explore the utility of a full combination of optical and NIR data to more precisely measure and correct for reddening in A. S. Friedman et al. (2008, in preparation).

Although ground-based NIR data can be obtained for lowredshift objects, rest-frame NIR observations for high-redshift $\mathrm{SNe}$ Ia will have to be done from space. Currently, rest-frame SN Ia Hubble diagrams of high-redshift SNe Ia have yet to be constructed beyond the $I$ band (Freedman 2005; Nobili et al. 2005), with limited studies of SNe Ia and their host galaxies conducted in the mid-infrared with Spitzer (Chary et al. 2005; Gerardy et al. 2007).

Because nearby SNe Ia are excellent standard candles in the NIR, it may be worth careful consideration of the rest-frame NIR for the space missions using SNe Ia for cosmology. For example, the SNAP (Aldering 2004) and DESTINY satellites (Lauer 2005), candidates for the NASA/DOE Joint Dark Energy Mission (JDEM) mission, are both currently designed with detectors sensitive out to $1.7 \mu \mathrm{m}$, which will only detect restframe $H$-band light $(1.6 \mu \mathrm{m})$ out to $z \sim 0.1$. Only a detector capable of observing rest-frame $H$-band at $z \sim 0.5-2$ could take full advantage of Nature's gift to us of a superb standard candle in the rest-frame $H$-band. Such a detector would require sensitivity from 2 to $5 \mu \mathrm{m}$. We can look forward to work of this type with James Webb Space Telescope (JWST), just over the horizon. The astronomical community should discuss which aspects of a JDEM mission could best be carried out in the restframe NIR.

The authors thank the anonymous referee for detailed and thorough reports that substantially improved the clarity of this manuscript. The Peters Automated Infrared Imaging Telescope (PAIRITEL) is operated by the Smithsonian Astrophysical Observatory (SAO) and was made possible by a grant from the Harvard University Milton Fund, the camera loan from the University of Virginia, and the continued support of the SAO and the University of California, Berkeley. Partial support for PAIRITEL operations and this work comes from National Aeronomics and Space 
Administration (NASA) grant NNG06GH50G (PAIRITEL: Infrared Follow-up for Swift Transients). J. S. B. and his group are partially supported by a DOE SciDAC Program through the collaborative agreement DE-FC02-06ER41438. PAIRITEL support and processing is conducted under the auspices of a DOE SciDAC grant (DE-FC02-06ER41453), which provides support to J. S. B.'s group. J. S. B. thanks the Sloan Research Fellowship for partial support.

We gratefully made use of the NASA/IPAC Extragalactic Database (NED). This publication makes use of data products from the 2MASS Survey, funded by NASA and the National
Science Foundation (NSF). IAUC/CBET were useful. M. W. V. is funded by a grant from the NSF (AST 05-7475). A. S. F. acknowledges support from an NSF Graduate Research Fellowship and a NASA Graduate Research Program Fellowship. M. M. acknowledges support in part from a Miller Research Fellowship. A. S. F, R. P. K, M. M., and S. B. thank the Kavli Institute for Theoretical Physics, which is supported by the NSF through grant PHY05-51164. The Center for Astrophysics Supernova Program is supported in part by NSF grant AST 06-06772. C. B. acknowledges support from the Harvard Origins of Life Initiative.

\section{REFERENCES}

Aldering, G. 2004, in Wide-Field Imaging From Space (Berkeley: LBNL), 3 Astier, P., et al. 2006, A\&A, 447, 31

Barris, B. J., Tonry, J. L., Novicki, M. C., \& Wood-Vasey, W. M. 2005, AJ, 130,2272

Barris, B. J., et al. 2004, ApJ, 602, 571

Benetti, S., et al. 2004, MNRAS, 348, 261

Bertin, E. 2005, SWarp (Paris: IAP), http://terapix.iap.fr/rubrique.php?id rubrique $=49$

Blake, C. H., Latham, D. W., \& Bloom, J. S. 2007, in ASP Conf. Ser. 366, Transiting Extrapolar Planets Workshop, ed. C. Afonso, D. Weldrake, \& T. Henning (San Francisco: ASP), 87

Blondin, S., et al. 2006, AJ, 131, 1648

Bloom, J. S., et al. 2006a, ApJ, 638, 354 2006b, in ASP Conf. Ser. 351, Astronomical Data Analysis Software and Systems XV, ed. C. Gabriel, C. Arviset, D. Ponz, \& S. Enrique (San Francisco: ASP), 751

- 2007, ApJ, 654, 878

Bronder, T. J., et al. 2007, A\&A, 477, 717

Butler, N. R., et al. 2006, ApJ, 652, 1390

Candia, P., et al. 2003, PASP, 115, 277

Carpenter, J. M. 2001, AJ, 121, 2851

Chary, R., Dickinson, M. E., Teplitz, H. I., Pope, A., \& Ravindranath, S. 2005, ApJ, 635, 1022

Cohen, M., Wheaton, W. A., \& Megeath, S. T. 2003, AJ, 126, 1090

Conley, A., Carlberg, R. G., Guy, J., Howell, D. A., Jha, S., Riess, A. G., \& Sullivan, M. 2007, ApJ, 664, L13

Conley, A., et al. 2006, AJ, 132, 1707 2008, ApJ, 681, 482

Cutri, R. M., et al. 2003, 2MASS All Sky Catalog of Point Sources (Pasadena: IPAC), http://irsa.ipac.caltech.edu/applications/Gator

Di Paola, A., Larionov, V., Arkharov, A., Bernardi, F., Caratti o Garatti, A., Dolci, M., Di Carlo, E., \& Valentini, G. 2002, A\&A, 393, L21

Eisner, J. A., Hillenbrand, L. A., White, R. J., Bloom, J. S., Akeson, R. L., \& Blake, C. H. 2007, ApJ, 669, 1072

Elias, J. H., Frogel, J. A., Hackwell, J. A., \& Persson, S. E. 1981, ApJ, 251, L13

Elias, J. H., Matthews, K., Neugebauer, G., \& Persson, S. E. 1985, ApJ, 296, 379

Elias-Rosa, N., et al. 2006, MNRAS, 369, 1880 2008, MNRAS, 384, 107

Ellis, R. S., et al. 2008, ApJ, 674, 51

Filippenko, A. V., et al. 1992, AJ, 104, 1543

Foley, R. J., et al. 2008, ApJ, 684, 68

Freedman, W. L. (for the Carnegie Supernova Project). 2005, in ASP Conf. Ser. 339, Observing Dark Energy, ed. S. C. Wolff \& T. R. Lauer (San Francisco: ASP), 50

Freedman, W. L., et al. 2001, ApJ, 553, 47

Frogel, J. A., Gregory, B., Kawara, K., Laney, D., Phillips, M. M., Terndrup, D., Vrba, F., \& Whitford, A. E. 1987, ApJ, 315, L129

Fruchter, A. S., \& Hook, R. N. 2002, PASP, 114, 144

Garavini, G., et al. 2007, A\&A, 470, 411

Garg, A., et al. 2007, AJ, 133, 403

Gerardy, C. L., et al. 2007, ApJ, 661, 995

Goldhaber, G., et al. 2001, ApJ, 558, 359

Graham, J. R., Meikle, W. P. S., Longmore, A. J., \& Williams, P. M. 1988, ApJ, 333,743

Guy, J., Astier, P., Nobili, S., Regnault, N., \& Pain, R. 2005, A\&A, 443, 781 Guy, J., et al. 2007, A\&A, 466, 11

Hamuy, M., Phillips, M. M., Suntzeff, N. B., Schommer, R. A., Maza, J., Smith, R. C., Lira, P., \& Aviles, R. 1996, AJ, 112, 2438

Hamuy, M., et al. 2006, PASP, 118, 2

Hernandez, M., et al. 2000, MNRAS, 319, 223

Hook, I. M., et al. 2005, AJ, 130, 2788

Immler, S., et al. 2006, ApJ, 648, L119

Jha, S., Riess, A. G., \& Kirshner, R. P. 2007, ApJ, 659, 122

Jha, S., et al. 1999, ApJS, 125, 73 2006, AJ, 131, 527

Kasen, D. 2006, ApJ, 649, 939

Kirshner, R. P., Willner, S. P., Becklin, E. E., Neugebauer, G., \& Oke, J. B. 1973, ApJ, 180, L97

Knop, R. A., et al. 2003, ApJ, 598, 102

Kocevski, D., et al. 2007, ApJ, 663, 1180

Krisciunas, K., Hastings, N. C., Loomis, K., McMillan, R., Rest, A., Riess, A. G., \& Stubbs, C. 2000, ApJ, 539, 658

Krisciunas, K., Phillips, M. M., \& Suntzeff, N. B. 2004a, ApJ, 602, L81

Krisciunas, K., Prieto, J. L., Garnavich, P. M., Riley, J.-L. G., Rest, A., Stubbs, C., \& McMillan, R. 2006, AJ, 131, 1639

Krisciunas, K., et al. 2001, AJ, 122, 1616

. 2003, AJ, 125, 166

. 2004b, AJ, 127, 1664

. $2004 \mathrm{c}, \mathrm{AJ}, 128,3034$

. 2005, AJ, 130, 350

. 2007, AJ, 133, 58

Kurtz, M. J., \& Mink, D. J. 1998, PASP, 110, 934

Lauer, T. R. 2005, NewA Rev., 49, 354

Leggett, S. K., et al. 2006, MNRAS, 373, 781

Leibundgut, B., et al. 1996, ApJ, 466, L21

Matheson, T., et al. 2008, AJ, 135, 1598

Meikle, W. P. S. 2000, MNRAS, 314, 782

Miknaitis, G., et al. 2007, ApJ, 666, 674

Modjaz, M. 2007, Ph.D. thesis, Harvard Univ.

Modjaz, M., et al. 2008, preprint (arXiv:0805.2201)

Mould, J. R., et al. 2000, ApJ, 529, 786

Neill, J. D., Hudson, M. J., \& Conley, A. 2007, ApJ, 661, L123

Nobili, S., et al. 2005, A\&A, 437, 789

Pastorello, A., et al. 2007a, MNRAS, 377, 1531 2007b, MNRAS, 376, 1301

Patat, F., Baade, D., Wang, L., Taubenberger, S., \& Wheeler, J. C. 2005, IAU Circ., 8631, 1

Perlmutter, S., et al. 1999, ApJ, 517, 565

. 1997, ApJ, 483, 565

Persson, S. E., Murphy, D. C., Krzeminski, W., Roth, M., \& Rieke, M. J. 1998, AJ, 116, 2475

Phillips, M. M., Lira, P., Suntzeff, N. B., Schommer, R. A., Hamuy, M., \&

Maza, J. 1999, AJ, 118, 1766

Phillips, M. M., et al. 2006, AJ, 131, 2615

2007, PASP, 119, 360

Pignata, G., et al. 2008, MNRAS, 388, 971

Prieto, J. L., Rest, A., \& Suntzeff, N. B. 2006, ApJ, 647, 501

Prieto, J. L., et al. 2007, preprint (arXiv:0706.4088)

Radburn-Smith, D. J., Lucey, J. R., \& Hudson, M. J. 2004, MNRAS, 355, 1378 Rest, A., et al. 2005, ApJ, 634, 1103

Riess, A. G., Press, W. H., \& Kirshner, R. P. 1996, ApJ, 473, 88

Riess, A. G., et al. 1998, AJ, 116, 1009 . 1999, AJ, 117, 707 2005, ApJ, 627, 579 2007, ApJ, 659, 98

Sahu, D. K., Tanaka, M., Anupama, G. C., Kawabata, K. S., Maeda, K., Tominaga, N., Nomoto, K., \& Mazzali, P. A. 2008, ApJ, 680, 580

Schechter, P. L., Mateo, M., \& Saha, A. 1993, PASP, 105, 1342

Skrutskie, M. F., et al. 2006, AJ, 131, 1163

Spergel, D. N., et al. 2007, ApJS, 170, 377 2003, ApJS, 148, 175

Stanishev, V., et al. 2007, A\&A, 469, 645 
Stritzinger, M., \& Sollerman, J. 2007, A\&A, 470, L1

Taubenberger, S., et al. 2008, MNRAS, 385, 75

Tominaga, N., et al. 2005, ApJ, 633, L97

Tonry, J. L., et al. 2003, ApJ, 594, 1

Valentini, G., et al. 2003, ApJ, 595, 779

Wang, L., Goldhaber, G., Aldering, G., \& Perlmutter, S. 2003, ApJ, 590, 944
Wang, L., Strovink, M., Conley, A., Goldhaber, G., Kowalski, M., Perlmutter, S., \& Siegrist, J. 2006, ApJ, 641, 50

Wang, X., et al. 2008, ApJ, 675, 626

Wood-Vasey, W. M., et al. 2007, ApJ, 666, 694

Yost, S. A., et al. 2006, ApJ, 636, 959 